\title{
Theoretical Study Of Compressible Flow Through Aneurysms
}

by

\author{
Maria Jumani \\ Master of Applied Mathematics, Karachi University, 2013
}

\author{
A thesis \\ presented to Ryerson University \\ in partial fulfillment of the \\ requirements for the degree of \\ Master of Science \\ Applied Mathematics
}

Toronto, Ontario, Canada, 2019

(C)Maria Jumani, 2019 


\section{AUTHOR'S DECLARATION FOR ELECTRONIC SUBMISSION OF A THESIS}

I hereby declare that I am the sole author of this thesis. This is a true copy of the thesis, including any required final revisions, as accepted by my examiners.

I authorize Ryerson University to lend this thesis to other institutions or individuals for the purpose of scholarly research.

I further authorize Ryerson University to reproduce this thesis by photocopying or by other means, in total or in part, at the request of other institutions or individuals for the purpose of scholarly research.

I understand that my thesis may be made electronically available to the public. 


\author{
Abstract \\ Theoretical Study Of Compressible Flow Through Aneurysms \\ Maria Jumani \\ Master of Science 2019 \\ Applied Mathematics \\ Ryerson University
}

The goal of this research is to analyze the effect of blood flow through expansions by using the KarmanPohlhausen method. The Karman-Pohlhausen method has previously been used in several research works to analyze the flow through constrictions. In this Thesis, the effect of different flow parameters (Reynolds number, compressibility, and slip) on pressure, pressure gradient, centerline velocity, and on wall shear stress are analyzed. Our results show that the pressure gradient curves are most affected by increasing Reynolds number and compressibility, as well as for smaller slip values $\left(w s_{0}\right)$. Furthermore, the scaled centerline velocity was least affected by varying Reynolds and Mach numbers, whereas changes are observed in centerline velocity curves for different slip values. The wall shear stress was essentially unchanged by the Reynolds numbers, compressibility range and slip values considered in this Thesis. 


\section{Acknowledgements}

I am grateful to Dr. Katrin Rohlf for her tremendous support and help and I am also grateful to Ryerson University for giving me this opportunity. 


\section{Dedication}

I would like to dedicate this Thesis to my father. 


\section{Table of Contents}

Author's Declaration $\quad$ ii

Abstract

Acknowledgements $\quad$ iv

Dedication $\quad$ v

List of Tables $\quad$ viii

List of Figures $\quad$ ix

List of Appendices $\quad x$

1 Introduction $\quad 1$

2 Introduction to Fluid Dynamics $\quad 3$

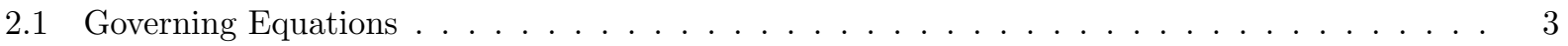

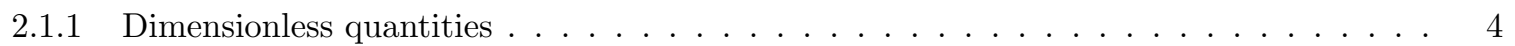

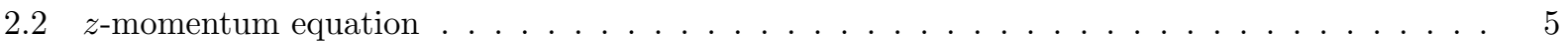

3 Karman-Pohlhausen method $\quad 9$

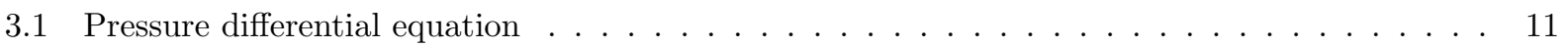

3.2 Upstream Properties . . . . . . . . . . . . . . . . . . . . . . . . . 12

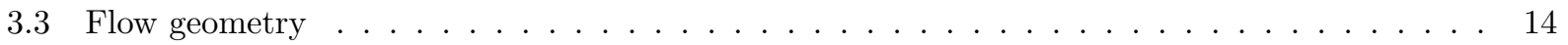

4 Numerical Results $\quad 16$

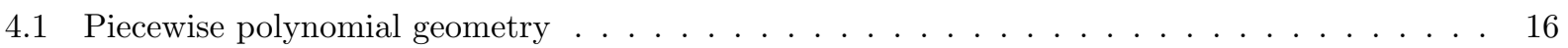

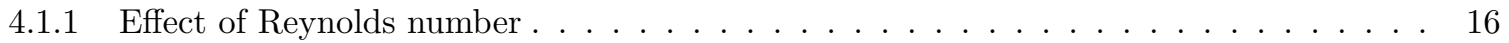

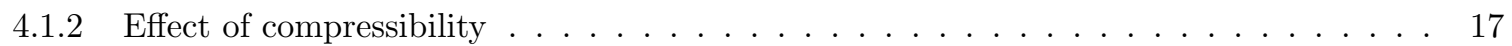

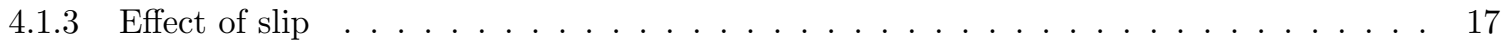

5 Conclusion and Future work $\quad 28$ 
Appendix A

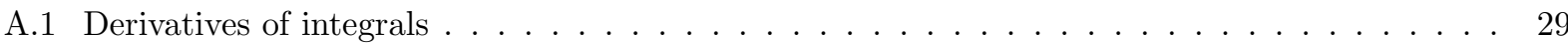

$\begin{array}{ll}\text { Appendix B } & 31\end{array}$

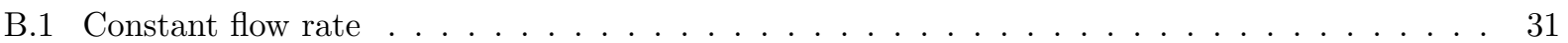

$\begin{array}{ll}\text { Appendix C } & 33\end{array}$

$\begin{array}{ll}\text { References } & 37\end{array}$ 


\section{List of Tables}

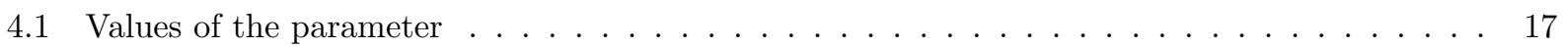




\section{List of Figures}

3.1 Piecewise polynomial flow geometry $\ldots \ldots \ldots \ldots \ldots \ldots \ldots$

4.1 Pressure curves in piecewise polynomial geometry with $w_{s}^{0}=0.04, l_{1}=4, l_{2}=1, R_{0}=0.1$, $\delta=0.05, M a_{0}=0.1$ for various Reynolds numbers $\ldots \ldots \ldots \ldots \ldots$

4.2 Pressure gradient curves in piecewise polynomial geometry with $w_{s}^{0}=0.04, l_{1}=4, l_{2}=1$, $R_{0}=0.1, \delta=0.05, M a_{0}=0.1$ for various Reynolds numbers $\ldots \ldots \ldots \ldots$

4.3 Centerline velocity curves in piecewise polynomial geometry with $w_{s}^{0}=0.04, l_{1}=4, l_{2}=1$, $R_{0}=0.1, \delta=0.05, M a_{0}=0.1$ for various Reynolds numbers $\ldots \ldots \ldots \ldots$

4.4 Wall shear stress curves in piecewise polynomial geometry with $w_{s}^{0}=0.04, l_{1}=4, l_{2}=1$, $R_{0}=0.1, \delta=0.05, M a_{0}=0.1$ for various Reynolds numbers $\ldots \ldots \ldots \ldots \ldots \ldots$

4.5 Pressure curves in piecewise polynomial geometry with $w_{s}^{0}=0.04, l_{1}=4, l_{2}=1, R_{0}=0.1$, $\delta=0.05, R e_{0}=200$ for various Mach numbers $\ldots \ldots \ldots \ldots \ldots \ldots \ldots \ldots \ldots \ldots \ldots \ldots$

4.6 Pressure gradient curves in piecewise polynomial geometry with $w_{s}^{0}=0.04, l_{1}=4, l_{2}=1$, $R_{0}=0.1, \delta=0.05, R e_{0}=200$ for various Mach numbers $\ldots \ldots \ldots \ldots \ldots \ldots$

4.7 Pressure curves in piecewise polynomial geometry with $R e_{0}=200, l_{1}=4, l_{2}=1, R_{0}=0.1$,

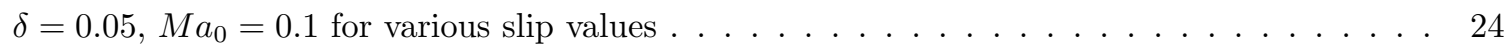

4.8 Pressure gradient curves in piecewise polynomial geometry with $R e_{0}=200, l_{1}=4, l_{2}=1$,

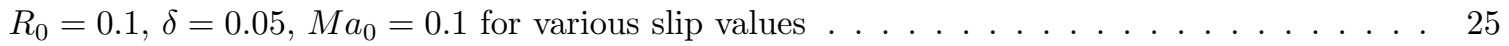

4.9 Scaled centerline velocity curves in piecewise polynomial geometry with $R e_{0}=200, l_{1}=4$, $l_{2}=1, R_{0}=0.1, \delta=0.05, M a_{0}=0.1$ for various slip values $\ldots \ldots \ldots \ldots$

4.10 Wall shear stress curves in piece-wise polynomial geometry with $R e_{0}=200, l_{1}=4, l_{2}=1$, $R_{0}=0.1, \delta=0.05, M a_{0}=0.1$ for various slip values $\ldots \ldots \ldots \ldots \ldots \ldots \ldots \ldots$ 


\section{List of Appendices}

$\begin{array}{ll}\text { Appendix A } & \mathbf{2 9}\end{array}$

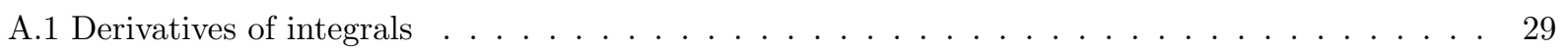

$\begin{array}{ll}\text { Appendix B } & 31\end{array}$

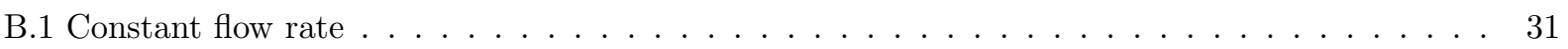

$\begin{array}{ll}\text { Appendix C } & 33\end{array}$ 


\section{Chapter 1}

\section{Introduction}

A blood vessel that at some point bulges out like a balloon is said to have an aneurysm. Aneurysms often occur at a weak spot along an artery. Certain factors are known to contribute to the formation of different kinds of aneurysms. It has been reported in the literature that smoking, hypertension and male sex are strong risk factors for the development of abdominal aortic aneurysms (AAAs). At the same time, there is no significance in increased growth rate of aneurysm with hypertension [29].

Subarachnoid hemorrhage (SAH) is a type of stroke that can be caused by the rupture of an intracranial aneurysm [28]. Smoking, hypertension, and excessive alcohol consumption are important risk factors known to increase the risk of subarachnoid hemorrhage (SAH) [13]. In describing the risk for aneurysm rupture, the location and type of aneurysm are important considerations [11]. "The mortality rate of ruptured abdominal aortic aneurysms (AAAs) is up to $75 \%$, making it the 13th leading cause of death in the US" [20]. Noninvasive techniques are presented in the literature for determining the rupture risk of Abdominal aortic aneurysm (AAA) [14].

In most research studies blood is treated as an incompressible fluid and no-slip boundary conditions are applied. In several studies wall slip has been applied to blood [1, 2, 10, 17, 23, 24, 27]. Furthermore, Particle-based methods have often been used to study the blood flow application [1, 2, 7, 35], that have compressibility effects built-in.

Much research has been done investigating the flow through aneurysms. Theoretical, numerical, and experimental methods can be used to analyze the impact of blood flow through the local expansions. One such work has been done by [21] numerically, where the flow was steady and incompressible with variable viscosity. The effect of nanoparticles on blood flowing through an artery with a combination of stenosis (narrowing of a blood vessel) and aneurysm was studied theoretically and numerically in [34]. The Lattice Boltzmann method was used to asses the influence of the asymmetry on the hemodynamics in a stented fusiform aneurysm [30]. Studies have been done on blood vessels with a severe stenosis and severe aneurysm to monitor the behavior of blood-flow in the diseased artery [3]. A particle-based method (multiparticle collision dynamics) was used to study blood flow through a local expansion in [25]. The effect of changing the severity of the aneurysm with slip (non-zero velocity component at the wall) as well as no-slip (zero velocity component at the wall) boundary conditions were considered for a range of low Reynolds numbers. Blood flow 
through an aneurysm during the cardiac cycle was studied by [32] numerically using a mixed Euler-Lagrange formulation. Investigation of blood flow through expansion has been done numerically $[12,16,19,31,33]$ as well as experimentally $[4,5,6,8]$. Several numerical experiments were carried out to understand the effect of the presence of the aneurysm, influence of the increase and decrease in pressure, and effect of the presence of multiple aneurysm on the flow features [18].

In this research the Karman-Pohlhausen method is used to study the flow through aneurysms analytically. This method has been used by many researchers in order to study the flow through stenosis. Forrester and Young [15] introduced the theoretical assesment for incompressible no-slip flow. The Karmann-Pohlhausen method was later used in $[9,22,26]$ to study the flow of micropolar fluid, of a couple stress fluid, and of a power law fluid respectively. Flow is considered as incompressible, and no-slip boundary conditions are applied in all of these studies. The Karman-Pohlhausen was later used in [1] and [27] to develop the axial velocity distribution for steady, Newtonian, weakly compressible flow through a constricted cylinder.

The goal of this research is to study the changes in pressure, pressure gradient, centerline velocity and wall shear stress of blood flow through mild expansions for various Reynolds and Mach numbers with slip boundary conditions. Blood is considered as a Newtonian, weakly compressible fluid. In our analysis the blood vessel is considered as a cylindrical tube with a local expansion commonly referred to as an aneurysm. A piecewise polynomial geometry is considered that is helpful in varying the location of maximum expansion allowing for asymmetric expansions.

This Thesis is organised as follows: In Chapter 2 we provide the Fluid Dynamics background that is the foundation of our analytical results, as well as the derivation of the integrated momentum equation. In Chapter 3 the analytical method that is used in our research, the pressure gradient equation, the flow geometry, and upstream properties are discussed. Chapter 4 contains the analysis of our numerical results. Finally, Chapter 5 provides important conclusions and future work. 


\section{Chapter 2}

\section{Introduction to Fluid Dynamics}

\subsection{Governing Equations}

The governing equations of motion for a compressible, isothermal, forced, steady flow with constant viscosity are

$$
\begin{aligned}
& \nabla \cdot(\rho \mathbf{u})=0 \text { (cons. mass) } \\
& \rho \mathbf{u} \cdot \nabla \mathbf{u}=-\nabla P+\rho \mathbf{f}+\mu \nabla^{2} \mathbf{u}+\frac{\mu}{3} \nabla(\nabla \cdot \mathbf{u}) \quad(\text { cons. momentum) }
\end{aligned}
$$

where $\nabla$ is the gradient operator, $\rho$ is the density, $\mathbf{u}$ is the velocity vector, $P$ is the pressure, $\mathbf{f}$ is the external force, and $\mu$ is the viscosity that is constant. The system also requires an equation of state for compressible flow, which, for an ideal gas, gives the pressure-density relationship

$$
P=\frac{k_{B} T}{m} \rho \text { (equation of state) }
$$

where $k_{B}$ is the Boltzmann constant, $T$ is the system temperature that is constant, and the mass of a fluid particle is $m$.

As we are dealing with axisymmetric flow, the governing equations can be written in cylindrical coordinates together with the assumed velocity vector

$$
\mathbf{u}=\left(u_{r}, u_{\theta}, u_{z}\right)=(u(r, z), 0, w(r, z)) .
$$

Since the velocity vector is a function of $r$ and $z$ only, so are the pressure and density. Here $r$ is the radial coordinate and $z$ is the axis of symmetry, and there is no flow in the $\theta$ direction.

An external force is acting in the $z$ direction only that is responsible for the flow. Thus

$$
\mathbf{f}=(0,0, \rho g)
$$


where $g$ is an acceleration constant. This forcing serves to be able to make a connection to particle-based simulations in future extension of this work. Now one can write the governing equations in cylindrical coordinates as follows

$$
\begin{array}{cc}
\frac{\partial}{\partial r}(\rho u)+\frac{\partial}{\partial z}(\rho w)+\frac{\rho u}{r}=0 & (\text { mass }) \\
\rho\left(u \frac{\partial u}{\partial r}+w \frac{\partial u}{\partial z}\right)=-\frac{\partial P}{\partial r} & (r \text {-momentum) } \\
+\mu\left(\frac{\partial^{2} u}{\partial r^{2}}+\frac{1}{r} \frac{\partial u}{\partial r}+\frac{\partial^{2} u}{\partial z^{2}}-\frac{u}{r^{2}}\right)+\frac{\mu}{3} \frac{\partial}{\partial r}(\nabla \cdot \mathbf{u}) \\
\rho\left(u \frac{\partial w}{\partial r}+w \frac{\partial w}{\partial z}\right)=\rho g-\frac{\partial P}{\partial z} & (z \text {-momentum }) \\
+\mu\left(\frac{\partial^{2} w}{\partial r^{2}}+\frac{1}{r} \frac{\partial w}{\partial r}+\frac{\partial^{2} w}{\partial z^{2}}\right)+\frac{\mu}{3} \frac{\partial}{\partial z}(\nabla \cdot \mathbf{u}) & \quad(\text { equation of state) }
\end{array}
$$

where

$$
\nabla \cdot \mathbf{u}=\frac{u}{r}+\frac{\partial u}{\partial r}+\frac{\partial w}{\partial z} .
$$

The $\theta$-momentum equation is identically satisfied.

In the next section, the differential equation for the pressure is derived by using these equations, subject to imposing slip at the wall. Specifically, $\mathbf{u} \cdot \mathbf{t}=w_{s}$ and $\mathbf{u} \cdot \mathbf{n}=0$, where $\mathbf{t}$ and $\mathbf{n}$ are the unit tangent and normal directions on the expanded cylinder wall; $w_{s}$ is a constant slip value. In the case of an axisymmetric flow with the wall described by $R=R(z)$, these equations can be solved to produce

$$
\left.u\right|_{r=R}=\frac{w_{s} R^{\prime}}{\sqrt{1+R^{\prime 2}}} \text { and }\left.w\right|_{r=R}=\frac{w_{s}}{\sqrt{1+R^{\prime 2}}}
$$

where $R^{\prime}=\frac{d R}{d z}$.

\subsubsection{Dimensionless quantities}

In this research some dimensionless quantities are used namely the Reynolds number and Mach number. The Reynolds number is defined as the ratio of an inertial term to viscous term. Reynolds numbers are helpful in predicting the flow pattern, whereas Mach numbers are used to measure the compressibility of the flow. These quantities are defined as follows,

$$
\begin{aligned}
R e & =\frac{\rho \bar{W} R}{\mu} \text { (Reynolds number) } \\
M a & =\frac{\bar{W}}{\sqrt{\frac{k_{B} T}{m}}} \text { (Mach number) }
\end{aligned}
$$


where $\rho$ is the density, $\bar{W}$ is the average velocity, $\mu$ is the constant viscosity, $k_{B}$ is the Boltzmann constant, $T$ is the constant system temperature and $m$ is mass the of the fluid particle.

\subsection{Derivation of integrated $z$-momentum equation}

As we are also dealing with axisymmetric flow, the analysis that was made for mild constrictions in Forrester and Young [15] is also applicable in our case for a mild expansion. This results in the approximation of the pressure as

$$
\frac{\partial P}{\partial r}=0
$$

As pressure is assumed independent of $r$, that means it is a function of $z$ only i.e $P=P(z)$, and through the equation of state, $\rho=\rho(z)$. After rearranging the equation of conservation of mass with this, it implies that

$$
\frac{\partial u}{\partial r}+\frac{u}{r}=-\frac{1}{\rho} \frac{\partial}{\partial z}(\rho w)
$$

Substituting the above equation in the $z$-momentum equation, together with the assumption that is suggested in Forrester and Young that $u \frac{\partial w}{\partial r} \ll w \frac{\partial w}{\partial z}$, we get

$$
\rho w \frac{\partial w}{\partial z}=\rho g-\frac{d P}{d z}+\mu\left(\frac{\partial^{2} w}{\partial r^{2}}+\frac{1}{r} \frac{\partial w}{\partial r}+\frac{4}{3} \frac{\partial^{2} w}{\partial z^{2}}\right)-\frac{\mu}{3} \frac{\partial}{\partial z}\left(\frac{1}{\rho} \frac{\partial}{\partial z}(\rho w)\right) .
$$

The assumption that was made in Forrester and Young says that the velocity of the flow in the radial direction $r$ is negligible as compared to the flow in the $z$ direction, as well as change in the flow due to change in $r$ is negligible as compared to the change in flow due to changes in $z$. On the basis of that assumption the term $u \frac{\partial w}{\partial r}$ is neglected. Multiplying equation (2.16) with $\frac{r}{\rho}$, and integrating with respect to $r$ from 0 to $R$, we get

$$
\begin{gathered}
\int_{0}^{R} r w \frac{\partial w}{\partial z} d r=\int_{0}^{R} r g d r-\int_{0}^{R} \frac{r}{\rho} \frac{d P}{d z} d r+\int_{0}^{R} \frac{\mu}{\rho} r\left(\frac{\partial^{2} w}{\partial r^{2}}+\frac{1}{r} \frac{\partial w}{\partial r}+\frac{4}{3} \frac{\partial^{2} w}{\partial z^{2}}\right) d r \\
-\int_{0}^{R} \frac{\mu}{3 \rho} r \frac{\partial}{\partial z}\left(\frac{1}{\rho} \frac{\partial}{\partial z}(\rho w)\right) d r
\end{gathered}
$$

which can be written as

$$
\begin{gathered}
\int_{0}^{R} r w \frac{\partial w}{\partial z} d r=\int_{0}^{R} r g d r-\int_{0}^{R} \frac{r}{\rho} \frac{d P}{d z} d r+\int_{0}^{R} \frac{\mu}{\rho} r\left(\frac{\partial^{2} w}{\partial r^{2}}+\frac{1}{r} \frac{\partial w}{\partial r}\right)+\int_{0}^{R}\left(\frac{4 \mu}{3 \rho} r \frac{\partial^{2} w}{\partial z^{2}}\right) d r \\
-\int_{0}^{R} \frac{\mu}{3 \rho} r \frac{\partial}{\partial z}\left(\frac{1}{\rho} \frac{\partial}{\partial z}(\rho w)\right) d r .
\end{gathered}
$$

After integrating the above equation and substituting $r\left(\frac{\partial^{2} w}{\partial r^{2}}+\frac{1}{r} \frac{\partial w}{\partial r}\right)=\frac{\partial}{\partial r}\left(r \frac{\partial w}{\partial r}\right)$, we get 


$$
\begin{aligned}
\frac{1}{2} \int_{0}^{R} r \frac{\partial}{\partial z} w^{2} d r=\frac{g R^{2}}{2} & -\frac{1}{\rho} \frac{d P}{d z} \frac{R^{2}}{2}+\left.\frac{\mu R}{\rho}\left(\frac{\partial w}{\partial r}\right)\right|_{r=R} \\
& +\int_{0}^{R} \frac{\mu}{\rho}\left[\frac{4}{3} r \frac{\partial^{2} w}{\partial z^{2}}-\frac{1}{3} r \frac{\partial}{\partial z}\left(\frac{1}{\rho} \frac{\partial}{\partial z}(\rho w)\right)\right] d r
\end{aligned}
$$

We assume that $\mu$ is constant, and found that $\rho=\rho(z)$. Thus the last term on the right-hand side of equation (2.17) can be written as

$$
r \frac{\partial}{\partial z}\left(\frac{1}{\rho} \frac{\partial}{\partial z}(\rho w)\right)=-\frac{1}{\rho^{2}} \frac{d \rho}{d z} \frac{\partial}{\partial z}(r \rho w)+\frac{1}{\rho} \frac{\partial^{2}}{\partial z^{2}}(r \rho w)
$$

Thus

$$
\begin{aligned}
\frac{1}{2} \int_{0}^{R} r \frac{\partial}{\partial z} w^{2} d r=\frac{g R^{2}}{2} & -\frac{1}{\rho} \frac{d P}{d z} \frac{R^{2}}{2}+\left.\frac{\mu R}{\rho}\left(\frac{\partial w}{\partial r}\right)\right|_{r=R} \\
& +\int_{0}^{R} \frac{\mu}{\rho}\left[\frac{4}{3} r \frac{\partial^{2} w}{\partial z^{2}}-\frac{1}{3}\left(-\frac{1}{\rho^{2}} \frac{d \rho}{d z} \frac{\partial}{\partial z}(r \rho w)+\frac{1}{\rho} \frac{\partial^{2}}{\partial z^{2}}(r \rho w)\right)\right] d r
\end{aligned}
$$

Next, we apply the Leibniz rule from Appendix A. The $z$-derivative can be taken outside of the integrals with respect to $r$, by using the Leibniz rule as follows (details are given in Appendix A):

$$
\begin{gathered}
\frac{1}{2} \int_{0}^{R} r \frac{\partial}{\partial z} w^{2} d r=\frac{1}{2} \frac{d}{d z} \int_{0}^{R} r w^{2} d r-\frac{1}{2} \frac{R R^{\prime} w_{s}^{2}}{\left(1+R^{\prime 2}\right)} \\
\frac{4}{3} \frac{\mu}{\rho} \int_{0}^{R} r \frac{\partial^{2} w}{\partial z^{2}} d r=\frac{4}{3} \frac{\mu}{\rho}\left[\frac{d^{2}}{d z^{2}} \int_{0}^{R} r w d r-\frac{R R^{\prime \prime} w_{s}}{\sqrt{1+R^{\prime 2}}}-\frac{R^{\prime 2} w_{s}}{\sqrt{1+R^{\prime 2}}}+\frac{2 R R^{\prime 2} R^{\prime \prime} w_{s}}{\left(1+R^{\prime 2}\right)^{\frac{3}{2}}}\right] \\
\int_{0}^{R} \frac{\partial^{2}}{\partial z^{2}}(\rho r w) d r=\frac{d^{2}}{d z^{2}} \int_{0}^{R} r \rho w d r-\frac{\rho\left(R R^{\prime \prime}+R^{\prime 2}\right) w_{s}}{\sqrt{1+R^{\prime 2}}}-\frac{2 R w_{s} R^{\prime}}{\sqrt{1+R^{\prime 2}} \frac{d \rho}{d z}+\frac{2 R \rho w_{s} R^{\prime 2} R^{\prime \prime}}{\left(1+R^{\prime 2}\right)^{3 / 2}}} \\
\frac{\mu}{3 \rho^{3}} \frac{d \rho}{d z}\left(\int_{0}^{R} \frac{\partial}{\partial z}(r w \rho) d r\right)=\frac{\mu}{3 \mu^{3}} \frac{d \rho}{d z}\left[\frac{d}{d z} \int_{0}^{R} r \rho w d r-\frac{R \rho w_{s}}{\sqrt{1+R^{\prime 2}}} R^{\prime}\right]
\end{gathered}
$$

Now equation (2.18) can be written as, 


$$
\begin{aligned}
\frac{1}{2} \frac{d}{d z} \int_{0}^{R} r w^{2} d r= & -\frac{1}{2} \frac{R R^{\prime} w_{s}^{2}}{\left(1+R^{\prime 2}\right)}-\frac{1}{\rho} \frac{d P}{d z} \frac{R^{2}}{2}+\frac{g R^{2}}{2}+\left.\frac{\mu R}{\rho}\left(\frac{\partial w}{\partial r}\right)\right|_{r=R} \\
& +\frac{4}{3} \frac{\mu}{\rho} \frac{d^{2}}{d z^{2}} \int_{0}^{R} r w d r-\frac{4}{3} \frac{\mu}{\rho} \frac{R w_{s} R^{\prime \prime}}{\sqrt{1+R^{\prime 2}}}-\frac{4}{3} \frac{\mu}{\rho} \frac{w_{s} R^{\prime 2}}{\sqrt{1+R^{\prime 2}}} \\
& +\frac{8}{3} \frac{\mu}{\rho} \frac{R R^{\prime 2} R^{\prime \prime} w_{s}}{\left(1+R^{\prime 2}\right)^{3 / 2}}-\frac{\mu}{3} \frac{1}{\rho^{2}}\left[\frac{d^{2}}{d z^{2}} \int_{0}^{R} r \rho w d r-\frac{\rho\left(R R^{\prime \prime}+R^{\prime 2}\right)}{\sqrt{1+R^{\prime 2}}} w_{s}\right. \\
& \left.-\frac{2 R w_{s} R^{\prime}}{\sqrt{1+R^{\prime 2}}} \frac{d \rho}{d z}+\frac{2 R \rho w_{s} R^{\prime 2} R^{\prime \prime}}{\left(1+R^{\prime 2}\right)^{3 / 2}}\right]+\frac{\mu}{3 \rho^{3}} \frac{d \rho}{d z}\left[\frac{d}{d z} \int_{0}^{R} r \rho w d r\right. \\
& \left.-\frac{R \rho w_{s} R^{\prime}}{\sqrt{1+R^{\prime 2}}}\right]
\end{aligned}
$$

which is the same as derived in [27], with constant viscosity. The above equation can be simplified by using the fact that the flow rate is constant. Flow rate $Q$ can be defined as follows,

$$
Q=\pi \rho R^{2} \bar{W}=2 \pi \rho \int_{0}^{R} r w(r, z) d r
$$

Recall that $\bar{W}$ is the average velocity. As the flow rate is constant (see Appendix B), the rate of change in the flow rate is zero which can be written as,

$$
\frac{d Q}{d z}=0
$$

Hence,

$$
\frac{d^{n}}{d z^{n}} \int_{0}^{R} r \rho w d r=0 \quad n=1,2
$$

and

$$
\frac{d}{d z} \int_{0}^{R} r w d r=-\frac{R^{2} \bar{W}}{2 \rho} \frac{d \rho}{d z}
$$

The detailed explanation is given in Appendix B. By using the above facts, equation (2.19) can be written 
as

$$
\begin{gathered}
\frac{1}{2} \frac{d}{d z} \int_{0}^{R} r w^{2} d r=-\frac{1}{2} \frac{R R^{\prime} w_{s}^{2}}{\left(1+R^{\prime 2}\right)}-\frac{k_{B} T}{m} \frac{1}{P} \frac{d P}{d z} \frac{R^{2}}{2}+\left.\frac{k_{B} T}{m} \frac{\mu R}{P}\left(\frac{\partial w}{\partial r}\right)\right|_{r=R} \\
+\frac{4}{3} \frac{k_{B} T}{m} \frac{\mu}{P} \frac{d^{2}}{d z^{2}} \int_{0}^{R} r w d r-\frac{4}{3} \frac{k_{B} T}{m} \frac{\mu}{P} \frac{R w_{s} R^{\prime \prime}}{\sqrt{1+R^{\prime 2}}}-\frac{4}{3} \frac{k_{B} T}{m} \frac{\mu}{P} \frac{w_{s} R^{\prime 2}}{\sqrt{1+R^{\prime 2}}} \\
-\frac{k_{B} T}{m} \frac{\mu}{3 P^{2}}\left[-\frac{P\left(R R^{\prime \prime}+R^{\prime 2}\right)}{\sqrt{1+R^{\prime 2}}} w_{s}-\frac{2 R w_{s} R^{\prime}}{\sqrt{1+R^{\prime 2}}} \frac{d P}{d z}+\frac{2 R P w_{s} R^{\prime 2} R^{\prime \prime}}{\left(1+R^{\prime 2}\right)^{3 / 2}}\right] \\
+\frac{8}{3} \frac{k_{B} T}{m} \frac{\mu}{P} \frac{R R^{\prime 2} R^{\prime \prime} w_{s}}{\left(1+R^{\prime 2}\right)^{3 / 2}}-\frac{\mu}{3 P^{2}} \frac{k_{B} T}{m} \frac{d P}{d z} \frac{R w_{s} R^{\prime}}{\sqrt{1+R^{\prime 2}}}+\frac{g R^{2}}{2}
\end{gathered}
$$

where the second derivative of the integral on the right-hand side of the equation (2.24) can be found by differentiating equation (2.23) as follows:

$$
\begin{aligned}
\frac{d^{2}}{d z^{2}} \int_{0}^{R} r w d r & =\frac{d}{d z}\left(-\frac{Q}{2 \pi \rho^{2}} \frac{d \rho}{d z}\right) \\
& =Q\left[\frac{1}{\pi \rho^{3}}\left(\frac{d \rho}{d z}\right)^{2}-\frac{1}{2 \pi \rho^{2}} \frac{d^{2} \rho}{d z^{2}}\right] \\
& =\rho R^{2} \bar{W}\left[\frac{1}{\rho^{3}}\left(\frac{d \rho}{d z}\right)^{2}-\frac{1}{2 \rho^{2}} \frac{d^{2} \rho}{d z^{2}}\right] \\
& =\frac{R^{2} \bar{W}}{\rho^{2}}\left(\frac{d \rho}{d z}\right)^{2}-\frac{R^{2} \bar{W}}{2 \rho} \frac{d^{2} \rho}{d z^{2}} .
\end{aligned}
$$

The density has been expressed in terms of pressure by using the equation of state (2.3) and we also used equation (2.24) with $\mathrm{n}=1$ and 2 resulting in the two integral terms on the right-hand side of the equation (2.19) becoming zero.

At this stage most terms in equation (2.24) are known flow parameters $\left(w_{s}, \frac{k_{B} T}{m}, \mu\right.$, flow rate $\left.\mathrm{Q}\right)$ or the given flow geometry $\left(R, R^{\prime}, R^{\prime \prime}\right)$ or the unknown terms (pressure or density) that need to be solved for, with the exception of the integral term on the left-hand side, and $\left.\left(\frac{\partial w}{\partial r}\right)\right|_{r=R}$. For the approximation of these two terms the Karman-Pohlhausen method is used which is described in the next chapter. 


\section{Chapter 3}

\section{Karman-Pohlhausen method}

Following $[1,15,27]$, the axial velocity $w$ is assumed to be a fourth-order polynomial that can be written in the form as follows,

$$
\frac{w(r, z)}{W}=A \eta+B \eta^{2}+C \eta^{3}+D \eta^{4}+E,
$$

where $\eta$ is the scaled radial coordinate which is defined as $\eta=\frac{R-r}{R}$ whose value is one at the center of the vessel and zero at the wall, and $W=W(z)$ is the centerline velocity that needs to be determined explicitly. The expressions for $A$ to $E$ are determined by imposing:

$$
w=\frac{w_{s}}{\sqrt{1+R^{\prime 2}}} \text { at } r=R \text { (slip boundary condition) }
$$

$$
\frac{\partial w}{\partial r}=0 \text { at } r=0 \text { (axisymmetric flow) }
$$

(iii) $\quad w=W$ at $r=0$ (definition of centerline velocity),

(iv) $\frac{\partial^{2} w}{\partial r^{2}}=-\frac{2\left(W-w_{s}\right)}{R^{2}}$ at $r=0$ (nearly parabolic flow with slip),

(v) $\frac{d P}{d z} \approx \rho g+\mu\left(\frac{\partial^{2} w}{\partial r^{2}}+\frac{1}{r} \frac{\partial w}{\partial r}\right)$ at $r=R$ ( using z-momentum equation). 
The values for the unknown constants can be found by imposing condition (i)-(v), resulting in

$$
\begin{aligned}
A & =\frac{1}{7}\left(-\lambda+10-12 E+T+2 \frac{w_{s}}{W}\right) \\
B & =\frac{1}{7}\left(3 \lambda+5-6 E-3 T+\frac{w_{s}}{W}\right) \\
C & =\frac{1}{7}\left(-3 \lambda-12+20 E+3 T-8 \frac{w_{s}}{W}\right) \\
D & =\frac{1}{7}\left(\lambda+4-9 E-T+5 \frac{w_{s}}{W}\right) \\
E & =\frac{w_{s}}{W \sqrt{1+R^{\prime 2}}}
\end{aligned}
$$

where $\lambda$ and $T$ are defined as,

$$
\lambda=\frac{R^{2}}{\mu W} \frac{d P}{d z} \quad \text { and } \quad T=\frac{\rho g R^{2}}{\mu W} .
$$

Now, we can evaluate $\left.\left(\frac{\partial w}{\partial r}\right)\right|_{r=R}$ by taking the derivative of the axial velocity. From equation (3.1)

$$
\begin{aligned}
\left.\frac{\partial w}{\partial r}\right|_{r=R} & =\left.\frac{d}{d r}\left[W\left(A \eta+B \eta^{2}+C \eta^{3}+D \eta^{4}+E\right)\right]\right|_{r=R} \\
& =\left.W\left[A \frac{d}{d r}\left(1-\frac{r}{R}\right)+B \frac{d}{d r}\left(1-\frac{r}{R}\right)^{2}+C \frac{d}{d r}\left(1-\frac{r}{R}\right)^{3}+D \frac{d}{d r}\left(1-\frac{r}{R}\right)^{4}+E \frac{d}{d r}(1)\right]\right|_{r=R} \\
& =\left.W\left[A\left(-\frac{1}{R}\right)+2 B\left(1-\frac{r}{R}\right)\left(-\frac{1}{R}\right)+3 C\left(1-\frac{r}{R}\right)^{2}\left(-\frac{1}{R}\right)+4 D\left(1-\frac{r}{R}\right)^{3}\left(-\frac{1}{R}\right)\right]\right|_{r=R} \\
& =-\frac{A W}{R}=-\frac{1}{7}\left(-\frac{R}{\mu} \frac{d P}{d z}+10 \frac{W}{R}-12 \frac{w_{s}}{R \sqrt{1+R^{\prime 2}}}+\frac{m}{k_{B} T} \frac{P g R}{\mu}+2 \frac{w_{s}}{R}\right) .
\end{aligned}
$$

Now, we can develop a relationship between the centerline velocity $W$ and the average velocity $\bar{W}$ by substituting equation (3.1) in equation (2.20). This gives

$$
Q=\pi \rho R^{2} \bar{W}=2 \pi \rho \int_{0}^{R} r W\left(A \eta+B \eta^{2}+C \eta^{3}+D \eta^{4}+E\right) d r .
$$

After integrating equation (3.8) and solving for $W$ in terms of $\bar{W}$, recalling that $W=W(z)$, we get

$$
\pi \rho R^{2} \bar{W}=\frac{-R^{4} \pi \rho}{105 \mu} \frac{d P}{d z}+\frac{97}{210} \pi \rho W R^{2}+\frac{17 \pi \rho R^{2}}{35} \frac{w_{s}}{\sqrt{1+R^{\prime 2}}}+\frac{R^{4}}{105} \frac{\rho g \pi}{\mu}+\frac{11 \pi \rho R^{2}}{210} w_{s}
$$

which can be rearranged to get

$$
W=\frac{2}{97} \frac{R^{2}}{\mu} \frac{d P}{d z}+\frac{210}{97} \bar{W}-\frac{102}{97} \frac{w_{s}}{\sqrt{1+R^{\prime 2}}}-\frac{2}{97} \frac{m}{k_{B} T} \frac{P g R^{2}}{\mu}-\frac{11}{97} w_{s} .
$$

Note that in the no-slip, and no force-driven case $\left(w_{s}=0, g=0\right)$, this relationship agrees with Forrester and Young. 


\subsection{Differential equation for pressure}

The differential equation for pressure can be obtained by substituting the expressions for $\frac{d}{d z} \int_{0}^{R} r w^{2} d r$ and $\left.\left(\frac{\partial w}{\partial r}\right)\right|_{z=R}$ into equation (2.24). The calculation for $\frac{d}{d z} \int_{0}^{R} r w^{2} d r$ is very tedious. Hence, Maple is used to evaluate the expression and the result is given in Appendix C. The obtained differential equation for the pressure can be written as

$$
\Omega_{1}\left(\frac{R^{3}}{\mu \bar{W}} \frac{d^{2} P}{d z^{2}}\right)+\Omega_{2}\left(\frac{R^{2}}{\mu \bar{W}} \frac{d P}{d z}\right)^{2}+\Omega_{3}\left(\frac{R^{2}}{\mu \bar{W}} \frac{d P}{d z}\right)+\Omega_{4}=0
$$

where, the coefficients $\Omega_{i}$ are

$$
\begin{aligned}
\Omega_{1}= & \frac{61}{1580712} R e \frac{R^{2}}{\mu \bar{W}} \frac{d P}{d z}+\frac{2}{3} \frac{M a^{2}}{R e}-\frac{899}{395178} \frac{w_{s} R e}{\bar{W}} \frac{1}{\sqrt{1+R^{\prime 2}}} \\
& -\frac{95}{790356} \frac{w_{s} R e}{\bar{W}}+\frac{631}{263452} R e-\frac{61}{1580712} \frac{g R}{W^{2}} R e^{2} \\
\Omega_{2}= & -\frac{4}{3} \frac{M a^{4}}{R e^{2}}+\frac{61}{526904} R^{\prime} R e-\frac{631}{263452} M a^{2}-\frac{61}{1580712} \frac{R g}{\bar{W}^{2}} M a^{2} R e \\
\Omega_{3}= & \frac{75}{194}-\frac{1}{3} \frac{w_{s}}{\bar{W}} \frac{R^{\prime} M a^{2}}{R e \sqrt{1+R^{\prime 2}}}-\frac{95}{197589} \frac{w_{s} R^{\prime} R e}{\bar{W}}-\frac{1798}{197589} \frac{w_{s} R^{\prime} R e}{\bar{W} \sqrt{1+R^{\prime 2}}} \\
& +\frac{899}{395178} \frac{w_{s} R R^{\prime} R^{\prime \prime} R e}{\bar{W}\left(1+R^{\prime 2}\right)^{3 / 2}}+\frac{631}{131726} R^{\prime} R e-\frac{61}{263452} \frac{R^{\prime} R e^{2} g R}{\bar{W}^{2}} \\
& +\frac{5125}{790356} \frac{w_{s}}{\bar{W}} M a^{2}+\frac{61}{1580712} \frac{g^{2} R^{2}}{\bar{W}^{4}} M a^{2} R e^{2}+\frac{95}{790356} \frac{w_{s} R g M a^{2} R e}{\bar{W}^{3}} \\
& +\frac{154937}{790356} \frac{w_{s}}{\bar{W} \sqrt{1+R^{\prime 2}} M a^{2}+\frac{899}{395178} \frac{w_{s} R g M a^{2} R e}{\bar{W}^{3} \sqrt{1+R^{\prime 2}}}-\frac{6610}{9409} M a^{2}}
\end{aligned}
$$




$$
\begin{aligned}
\Omega_{4}= & -\frac{75}{194} \frac{g R R e}{\bar{W}^{2}}+\frac{R^{\prime \prime} R w_{s}}{\sqrt{1+R^{\prime 2}} \bar{W}}+\frac{R^{\prime 2} w_{s}}{\sqrt{1+R^{\prime 2}} \bar{W}}+\frac{300}{97}+\frac{12}{97} \frac{w_{s}}{\bar{W}} \\
& -\frac{312}{97} \frac{w_{s}}{\bar{W}} \frac{1}{\sqrt{1+R^{\prime 2}}}+\frac{154937}{790356} \frac{R^{\prime} R^{\prime \prime} w_{s} R R e}{\left(1+R^{\prime 2}\right)^{3 / 2} \bar{W}}-\frac{2 R^{\prime \prime} R^{\prime 2} w_{s} R}{\left(1+R^{\prime 2}\right)^{3 / 2} \bar{W}} \\
& -\frac{611627}{1975890} \frac{R^{\prime} w_{s}^{2} R e}{\bar{W}^{2}\left(1+R^{\prime 2}\right)}-\frac{188159}{987945} \frac{R R^{\prime} R^{\prime \prime} w_{s}^{2} R e}{\bar{W}^{2}\left(1+R^{\prime 2}\right)^{2}} \\
& -\frac{899}{395178} \frac{g R w_{s}}{\bar{W}^{3}} \frac{R R^{\prime} R^{\prime \prime} R e^{2}}{\left(1+R^{\prime 2}\right)^{3 / 2}}+\frac{61}{526904} \frac{R^{\prime} g^{2} R^{2} R e^{3}}{\bar{W}^{4}} \\
& +\frac{149}{329315} \frac{R^{\prime} R^{\prime \prime} w_{s}^{2} R R e}{\left(1+R^{\prime 2}\right) \bar{W}^{2}}-\frac{149}{329315} \frac{R R^{\prime 3} R^{\prime \prime} w_{s}^{2} R e}{\bar{W}^{2}\left(1+R^{\prime 2}\right)^{2}} \\
& -\frac{23837}{3951780} \frac{R^{\prime} R^{\prime \prime} w_{s}^{2} R R e}{\left(1+R^{\prime 2}\right)^{3 / 2} \bar{W}^{2}}-\frac{631}{131726} \frac{R^{\prime} g R R e^{2}}{\bar{W}^{2}}-\frac{6610}{9409} R^{\prime} R e \\
& +\frac{23837}{1975890} \frac{R^{\prime} w_{s}^{2} R e}{{\sqrt{1+R^{\prime 2}} \bar{W}^{2}}^{2}}+\frac{1798}{197589} \frac{R^{\prime} g R w_{s} R e^{2}}{{\sqrt{1+R^{\prime 2}}}^{3}} \\
& +\frac{149}{329315} \frac{R^{\prime 3} w_{s}^{2} R e}{\left(1+R^{\prime 2}\right) \bar{W}^{2}}+\frac{95}{197589} \frac{R^{\prime} g R w_{s} R e^{2}}{\bar{W}^{3}}
\end{aligned}
$$

Note that the Mach number and Reynolds number are $z$-dependent expressions, as a result of the expansion and only constant in a cylinder of fixed cross section. Equation (3.11) is solved subject to initial conditions $P=P_{0}$ and $\frac{d \rho}{d z}=0$ at $z=0$.

\subsection{Upstream Properties}

The pressure differential equation presented in the previous section depends on $z$, and on dimensionless $z$-dependent numbers. By using the constant flow rate property, a few terms in the pressure differential equation can be replaced with constant upstream values. Constant flow rate (2.20) means

$$
\rho_{0} R_{0}^{2} \bar{W}_{0}=\rho R^{2} \bar{W}
$$

where zero subscript denote upstream values. Since

$$
R e_{0}=\frac{\rho_{0} \overline{W_{0}} R_{0}}{\mu},
$$

this then gives

$$
R e=\frac{\rho \bar{W} R}{\rho_{0} \overline{W_{0}} R_{0}} R e_{0}
$$

Now, by using equation (3.16), equation (3.18) can be written as,

$$
R e=\frac{R_{0}}{R} R e_{0}
$$


Similarly,

$$
\begin{aligned}
M a & =\frac{P_{0} R_{0}^{2}}{P R^{2}} M a_{0} \\
\frac{w_{s}}{\bar{W}} & =\frac{P R^{2}}{P_{0} R_{0}^{2}} \frac{w_{s}}{\bar{W}_{0}} \\
\frac{R g}{\bar{W}^{2}} & =\left(\frac{P}{P_{0}}\right)^{2}\left(\frac{R}{R_{0}}\right)^{5} \frac{R_{0} g}{\bar{W}_{0}^{2}}
\end{aligned}
$$

where $M a_{0}$ and $R e_{0}$ are the constant upstream Mach and Reynolds numbers respectively, $P_{0}$ is the upstream pressure and $\overline{W_{0}}$ is the average upstream velocity.

Additionally, the $r$-dependent axial velocity can be found by solving equation (2.8) upstream where $w=w(r)$, $\frac{d P}{d z}=0$ and $\rho=\rho_{0}$ are constant, subject to the boundary condition $w\left(r=R_{0}\right)=w_{s}$, and imposing that $w(r=0)$ is finite. The result is

$$
w(r)=\frac{\rho_{0} g}{4 \mu}\left(R_{0}^{2}-r^{2}\right)+w_{s}
$$

It follows that, upstream, the centerline velocity is

$$
W_{0}=w(r=0)=\frac{\rho_{0} g R_{0}^{2}}{4 \mu}+w_{s}
$$

and the average upstream velocity is

$$
\bar{W}_{0}=\frac{\int_{0}^{R_{0}} r w d r}{\int_{0}^{R_{0}} r d r}=\frac{\rho_{0} g R_{0}^{2}}{8 \mu}+w_{s} .
$$

Dividing equation (3.25) by $\bar{W}_{0}$, using equation (2.12) upstream, we get

$$
\begin{aligned}
1 & =\frac{1}{8} \frac{\rho_{0} \bar{W}_{0} R_{0}}{\mu} \frac{g R_{0}}{\bar{W}_{0}^{2}}+\frac{w_{s}}{\bar{W}_{0}} \\
1 & =\frac{1}{8} R e_{0} \frac{g R_{0}}{\bar{W}_{0}^{2}}+\frac{w_{s}}{\bar{W}_{0}} \\
R e_{0} & =\frac{8\left(1-w_{s}^{0}\right)}{g^{0}},
\end{aligned}
$$

where $w_{s}^{0}=\frac{w_{s}}{\bar{W}_{0}}$ and $g^{0}=\frac{g R_{0}}{\bar{W}_{0}^{2}}$ are dimensionless wall slip and forcing terms respectively scaled by (constant) upstream quantities. 


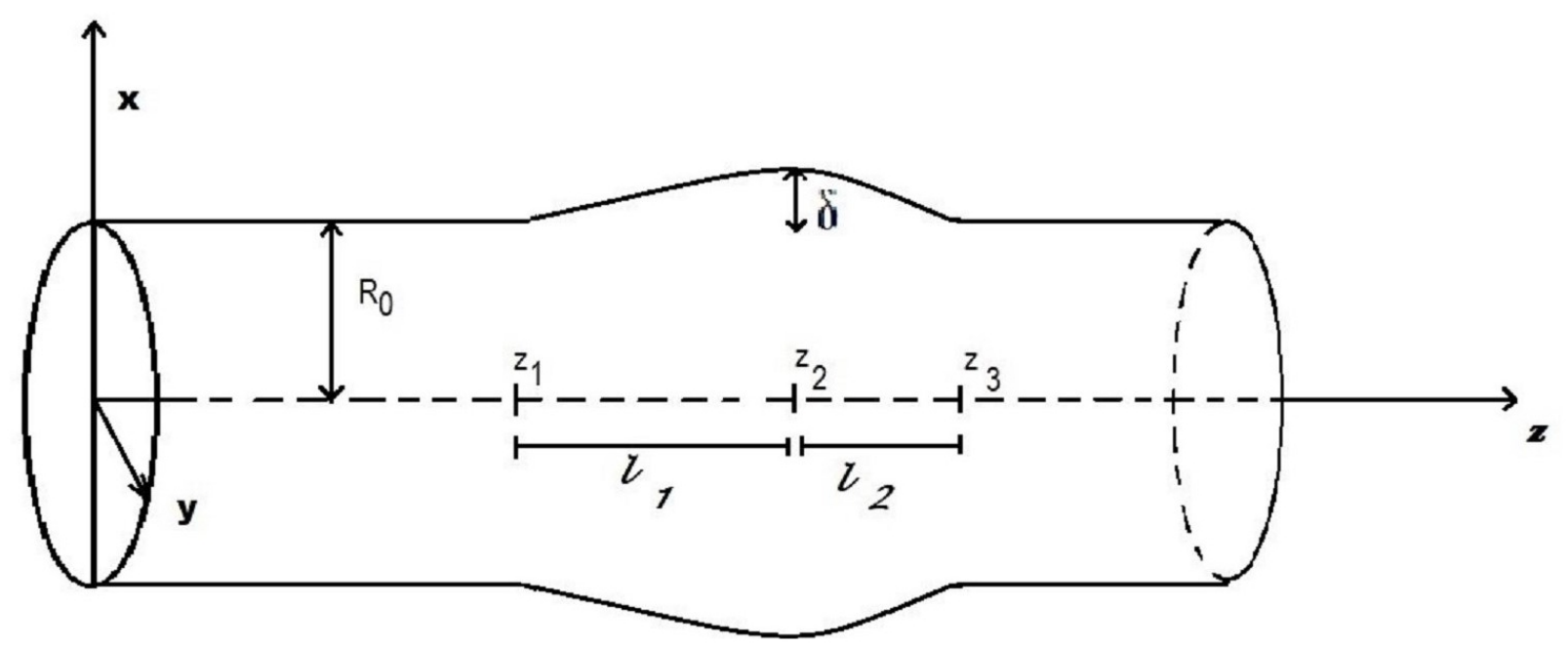

Figure 3.1: Piecewise polynomial flow geometry

\subsection{Flow geometry}

In the literature several axisymmetric flow geometries are considered for the physiologically meaningful study of the blood flow through an aneurysm. Here we consider the polynomial geometry that can be used to vary the location of maximum expansion, creating asymmetric scenarios. The following axisymmetric geometry is described by $R=R(z)$ :

$$
R(z)= \begin{cases}R_{0} & z \leq z_{1} \\ a z^{3}+b z^{2}+c z+d & z_{1} \leq z \leq z_{2} \\ e z^{3}+f z^{2}+g z+h & z_{2} \leq z \leq z_{3} \\ R_{0} & z \geq z_{3}\end{cases}
$$

where $z_{1}$ is the start of the aneurysm, $z_{2}$ is the location of maximum expansion, $z_{3}$ is the end of the aneurysm, $l_{1}+l_{2}$ determines the length of the expansions and $\delta$ controls the severity of the expansion (see figure 3.1). The expressions for $a-h$ are the same as outlined in $[1,27]$ with opposite sign for $\delta$. They are 


$$
\begin{aligned}
& a=-\frac{2 \delta}{l_{1}^{2}} \quad b=\frac{3 \delta\left(2 z_{1}+l_{1}\right)}{l_{1}{ }^{3}} \quad c=-\frac{6 \delta z_{1}\left(z_{1}+l_{1}\right)}{l_{1}{ }^{3}} \\
& d=\frac{2 \delta z_{1}{ }^{3}+3 \delta z_{1}{ }^{2} l_{1}+R_{o} l_{1}{ }^{3}}{l_{1}{ }^{3}} \quad e=\frac{2 \delta}{l_{2}{ }^{3}} \quad f=\frac{3\left(2 z_{1}+2 l_{1}+l_{2}\right) \delta}{l_{2}{ }^{3}} \\
& g=\frac{6 \delta\left(z_{1}{ }^{2}+2 z_{1} l_{1}+l_{1}{ }^{2}+z_{1} l_{2}+l_{1} L_{2}\right)}{l_{2}{ }^{3}} \\
& h=-\frac{3 \delta l_{1}{ }^{2} l_{2}+3 \delta z_{1}{ }^{2} l_{2}+6 \delta z_{1} l_{1} l_{2}+2 \delta l_{1}{ }^{3}+2 \delta z_{1}{ }^{3}+6 \delta z_{1}{ }^{2} l_{1}-R_{o} l_{2}{ }^{3}-\delta l_{2}{ }^{3}}{l_{2}{ }^{3}}
\end{aligned}
$$




\section{Chapter 4}

\section{Numerical Results}

\subsection{Piecewise polynomial geometry}

In this chapter, we plot the solution curves for various Reynolds numbers, compressibility and slip values, keeping the values of other parameters fixed such as $z_{1}=6 \mathrm{~m}, l_{1}=4 \mathrm{~m}, l_{2}=1 \mathrm{~m}, R_{0}=0.1 \mathrm{~m}, \delta=0.05 \mathrm{~m}$, $\rho_{0}=1050 \mathrm{~kg} / \mathrm{m}^{3}, \mu=0.0035 \mathrm{~kg} / \mathrm{ms}$, whereas the values for $\overline{W_{0}}, g_{0}$ and $\frac{k_{B} T}{m}$ are determined from the relations described below

$$
\overline{W_{0}}=\frac{R e_{0} \mu_{0}}{\rho_{0} R_{0}}, \quad \frac{k_{B} T}{m}=\frac{\overline{W_{0}^{2}}}{M a_{0}^{2}}, \quad g_{0}=\frac{8\left(1-w_{s}^{0}\right)}{R e_{0}} .
$$

The piecewise polynomial geometry is implemented from Akhter and Rohlf [1] and pressure, pressure gradient, centerline velocity and wall shear stress curves are analyzed. The Runge-Kutta method (RK4) is used to generate the numerical solution of the differential equation (3.11)-(3.15) in Matlab. The scaling factor for centerline velocity is $\frac{1}{2 \overline{W_{0}}-w_{s}^{0} \overline{W_{0}}}$, whereas wall shear stress is scaled by $\frac{-R_{0}}{\mu_{0} \overline{W_{0}}}$

Values used for the simulations are presented in Table 4.1. The upstream Reynolds, Mach and slip values $\left(R e_{0}, M a_{0}, w_{s} 0\right)$ are chosen, from which $\overline{W_{0}}, \frac{k_{B} T}{m}$ and $g_{0}$ are computed from (4.1). The results for all simulations are given in the Table 4.1.

\subsubsection{Effect of Reynolds number}

In order to study the effect of various Reynolds numbers on the blood flow through mild expansions we keep the Mach number fixed $\left(M a_{0}=0.1\right)$ and plot the solution curves for $R e_{0}=200, R e_{0}=300, R e_{0}=400$. Scaled pressure/density curves are presented in Figure 4.1 for weakly compressible flow with slip, $P_{0}$ is the upstream pressure obtained from the equation of state (2.3) upstream. These curves indicate that as the Reynolds number increases, pressure decreases and the largest dip is attained by the curve for the largest Reynolds number.

Pressure gradient curves are shown in Figure 4.2 for various Reynolds numbers. As we can see by glancing 
Table 4.1: Values of the parameter

\begin{tabular}{|c|c|c|c|c|c|}
\hline \hline$R e_{0}$ & $M a_{0}$ & $w_{s}^{0}$ & $\overline{W_{0}}$ & $\frac{k_{B} T}{m}$ & $g_{0}$ \\
\hline 200 & 0.1 & 0 & 0.006666666667 & 0.0044444444444 & 0.04 \\
200 & 0.1 & 0.04 & 0.006666666667 & 0.004444444444 & 0.0384 \\
200 & 0.1 & 0.1 & 0.006666666667 & 0.0044444444444 & 0.036 \\
200 & 0.1 & 0.3 & 0.006666666667 & 0.004444444444 & 0.028 \\
200 & 0.3 & 0.04 & 0.006666666667 & 0.000493827161 & 0.0384 \\
200 & 0.5 & 0.04 & 0.006666666667 & 0.00077777778 & 0.0384 \\
300 & 0.1 & 0.04 & 0.01 & 0.01 & 0.0256 \\
400 & 0.1 & 0.04 & 0.013333333333 & 0.017777777778 & 0.0192 \\
\hline
\end{tabular}

at the figure, larger minima/maxima are attained for larger Reynolds numbers, and there is no change in the pressure gradient in the upstream and downstream portion as a result of changes in the Reynolds number. In Figure 4.3, scaled centerline velocity curves are presented for various Reynolds numbers. No significant changes are observed in centerline velocity with the change in Reynolds numbers. Likewise the scaled wall shear curves in Figure 4.4 show little change as Reynolds number changes.

\subsubsection{Effect of compressibility}

Working with the same piecewise flow geometry discussed in the previous section, the solution curves are obtained for various $M a_{0}$ for fixed Reynolds number $(\mathrm{Re}=200)$. Figure 4.5 shows the effect of change in pressure due to change in compressibility (Mach numbers). As the Mach numbers increase, the pressure decreases, with maximum decrease in the pressure attained by the curve with $M a_{0}=0.5$. Minor changes are observed in the pressure gradient curves for $M a_{0}=0.1$ and $M a_{0}=0.3$ in Figure 4.6, whereas obvious differences are observed in the minima/maxima of the pressure gradient curve for $M a_{0}=0.5$. The centerline velocity and wall shear stress curves show little change while varying Mach numbers, and thus graphs are not presented here.

\subsubsection{Effect of slip}

In this section we analyze the solution curves for various slip values $\left(w_{s 0}=0, w_{s 0}=0.1, w_{s 0}=0.3\right)$ with $R e_{0}=200$ and $M a_{0}=0.1$. The minimum pressure is attained by the curve with no-slip $\left(w_{s 0}=0\right)$, and as the slip increases the pressure increases (see Figure 4.7).

Pressure gradient curves are shown in Figure 4.8 for various slip values. The largest minimum is attained by the curve with no-slip, whereas no significant changes are observed in the maxima of the curves due to varying slip values. Scaled centerline velocity curves are presented in Figure 4.9 for various slip values. It is observed that the centerline velocity decreases more with increasing slip. Wall shear stress curves are presented in Figure 4.10 for various slip values. No significant changes are observed in wall shear stress curves as slip increases. 


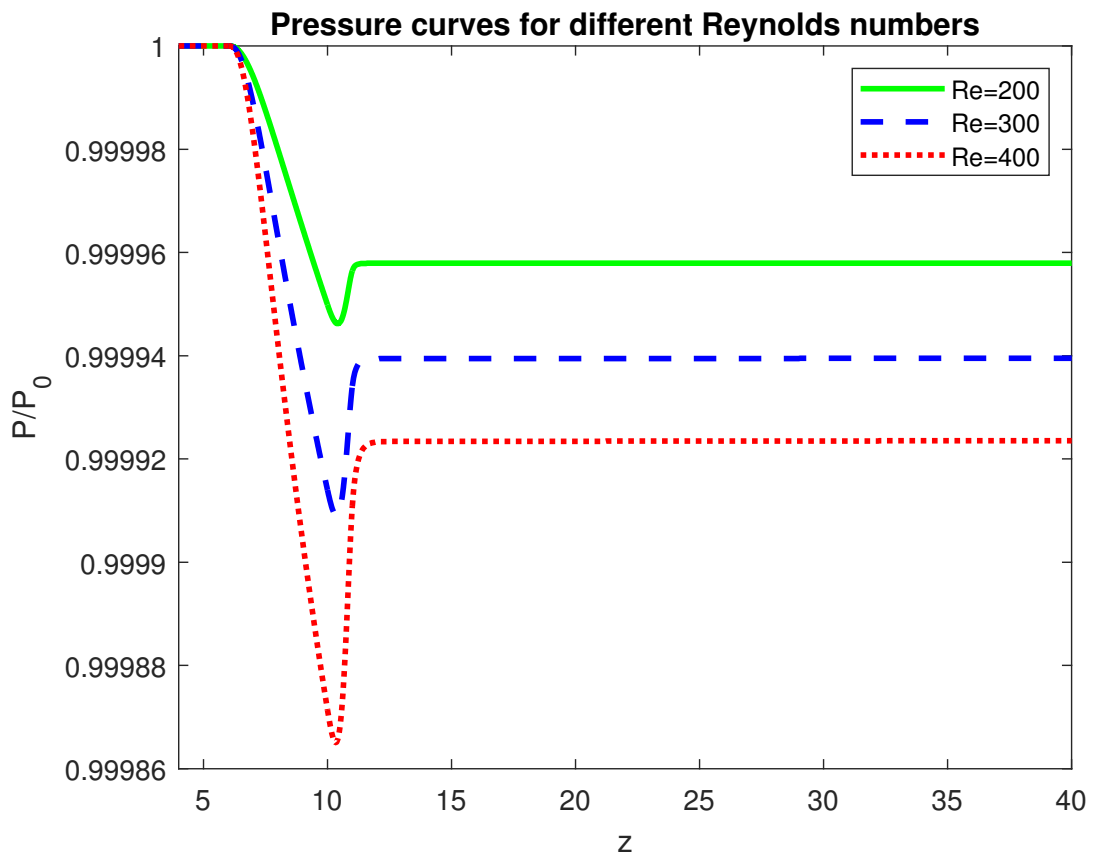

Figure 4.1: Pressure curves in piecewise polynomial geometry with $w_{s}^{0}=0.04, l_{1}=4, l_{2}=1, R_{0}=0.1$, $\delta=0.05, M a_{0}=0.1$ for various Reynolds numbers 


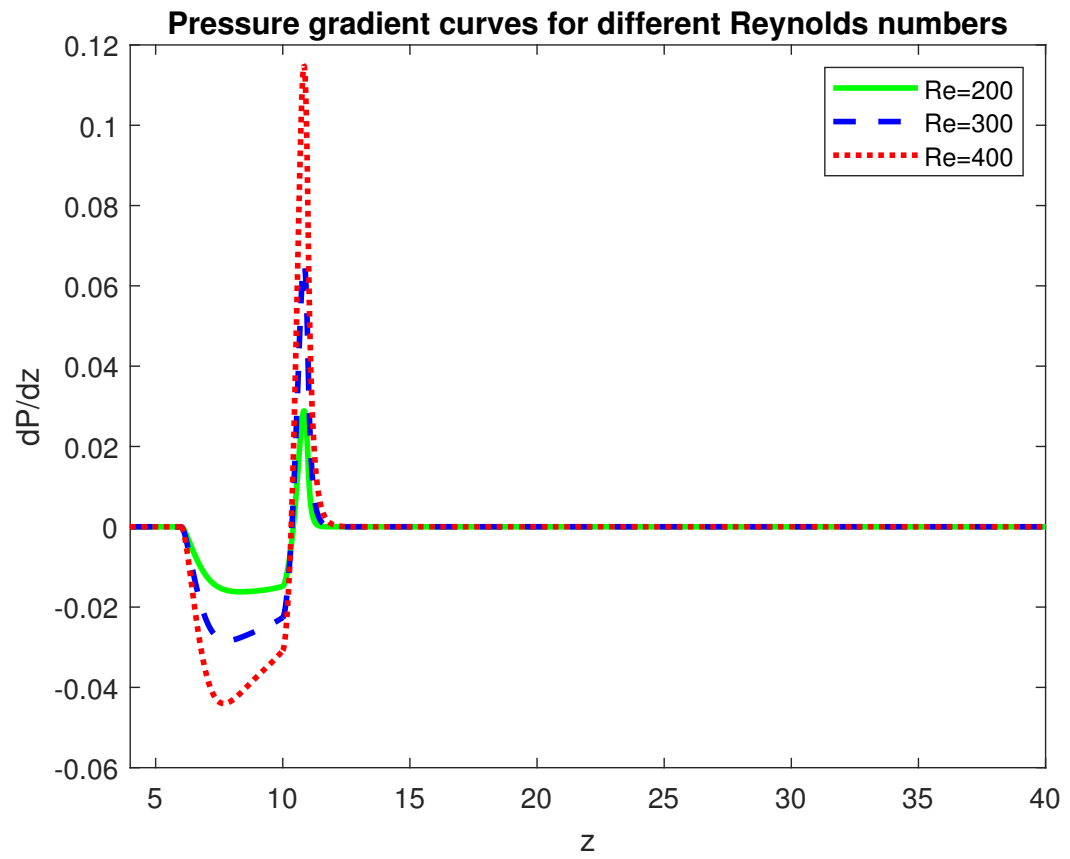

Figure 4.2: Pressure gradient curves in piecewise polynomial geometry with $w_{s}^{0}=0.04, l_{1}=4, l_{2}=1$, $R_{0}=0.1, \delta=0.05, M a_{0}=0.1$ for various Reynolds numbers 


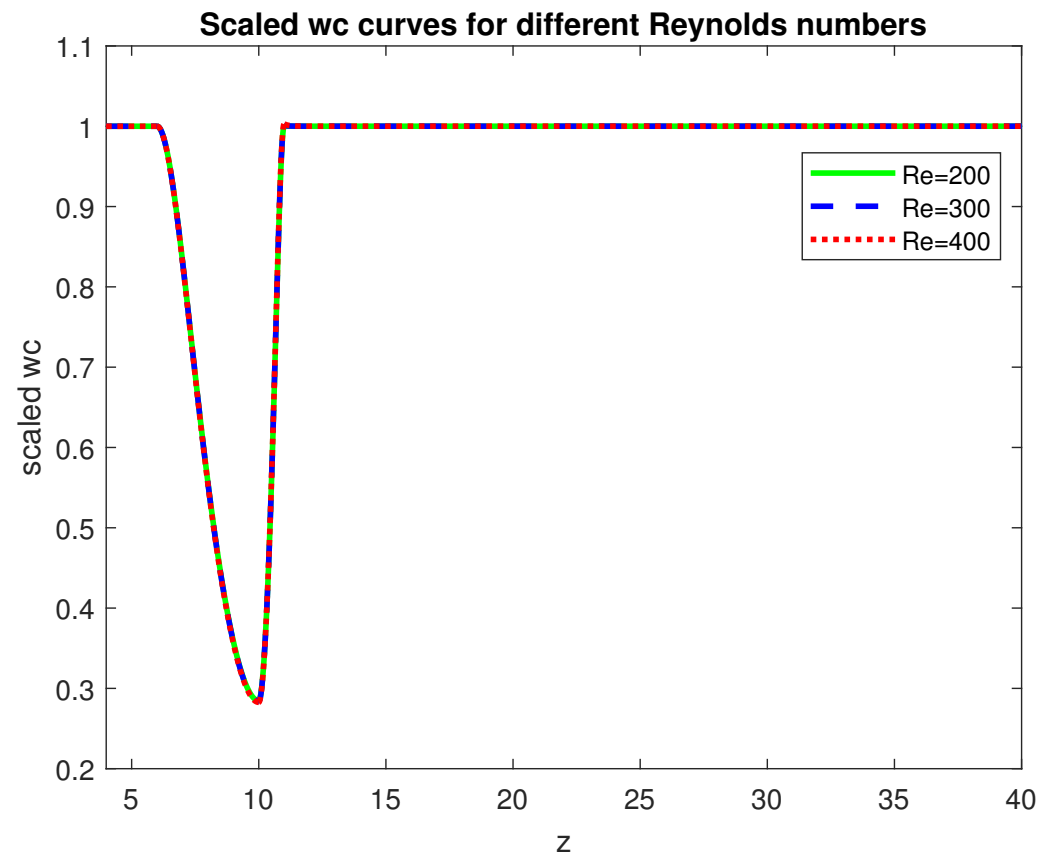

Figure 4.3: Centerline velocity curves in piecewise polynomial geometry with $w_{s}^{0}=0.04, l_{1}=4, l_{2}=1$, $R_{0}=0.1, \delta=0.05, M a_{0}=0.1$ for various Reynolds numbers 


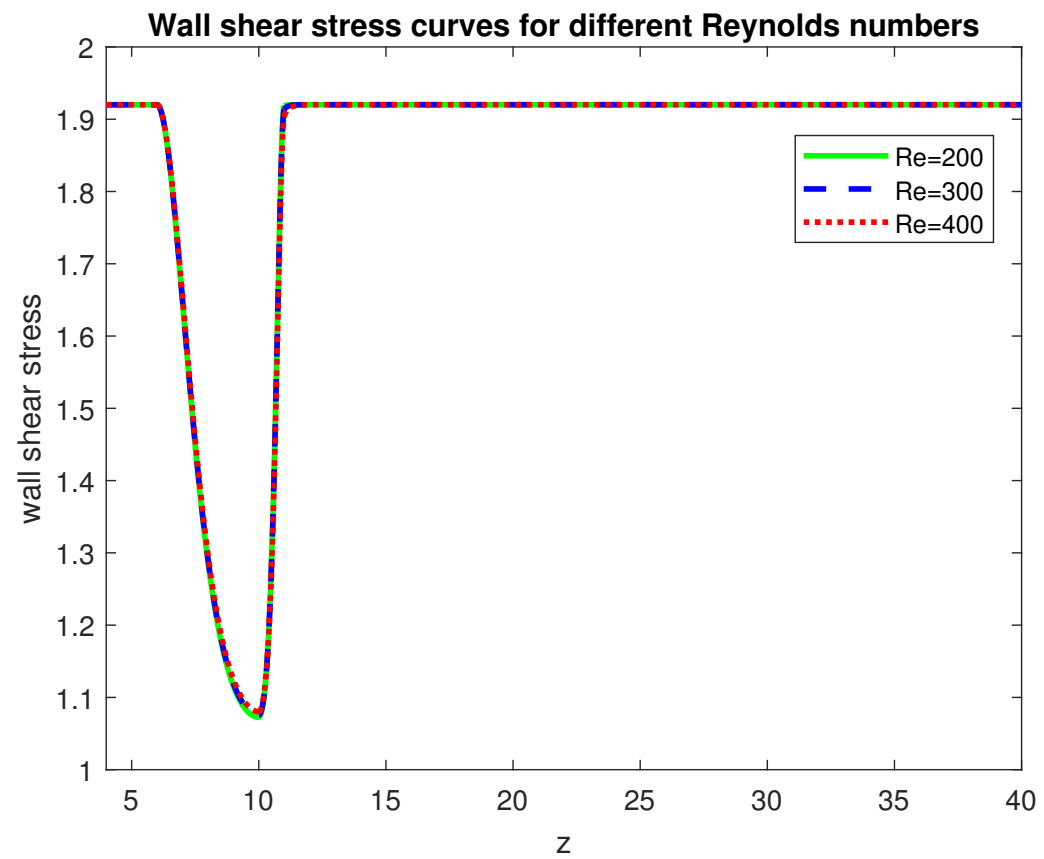

Figure 4.4: Wall shear stress curves in piecewise polynomial geometry with $w_{s}^{0}=0.04, l_{1}=4, l_{2}=1$, $R_{0}=0.1, \delta=0.05, M a_{0}=0.1$ for various Reynolds numbers 


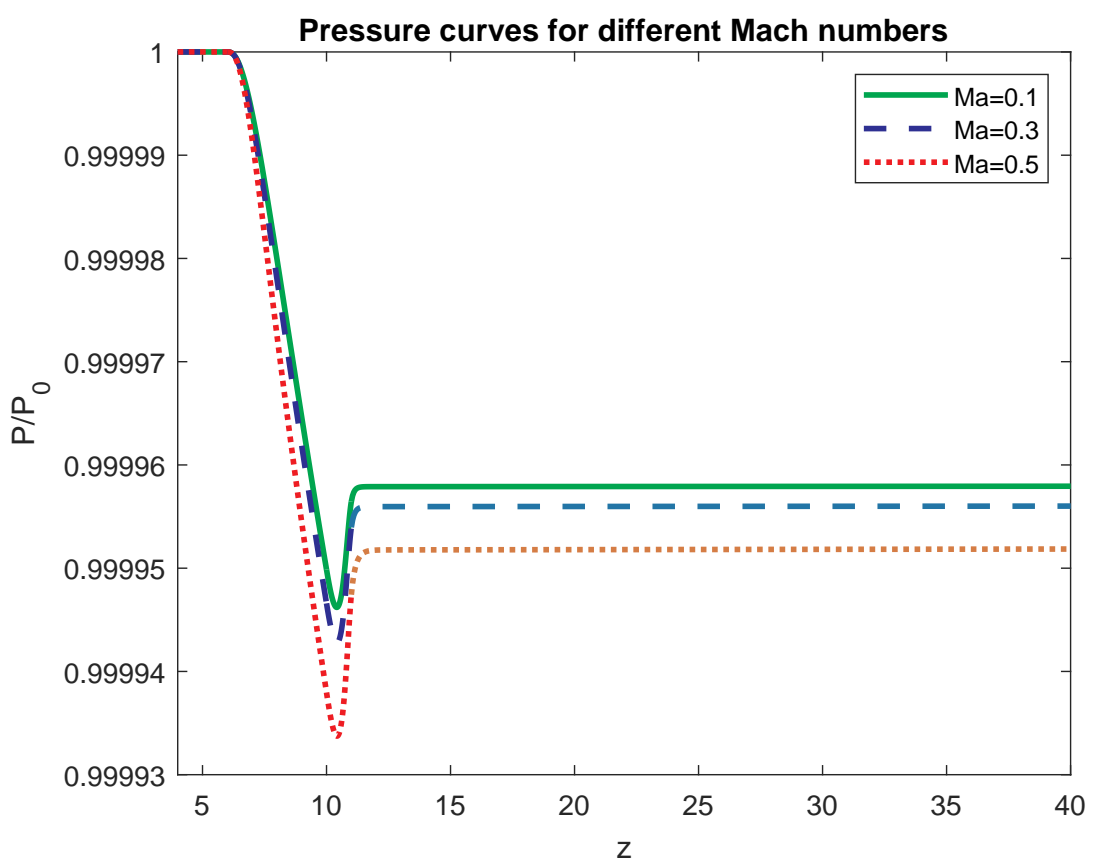

Figure 4.5: Pressure curves in piecewise polynomial geometry with $w_{s}^{0}=0.04, l_{1}=4, l_{2}=1, R_{0}=0.1$, $\delta=0.05, R e_{0}=200$ for various Mach numbers 


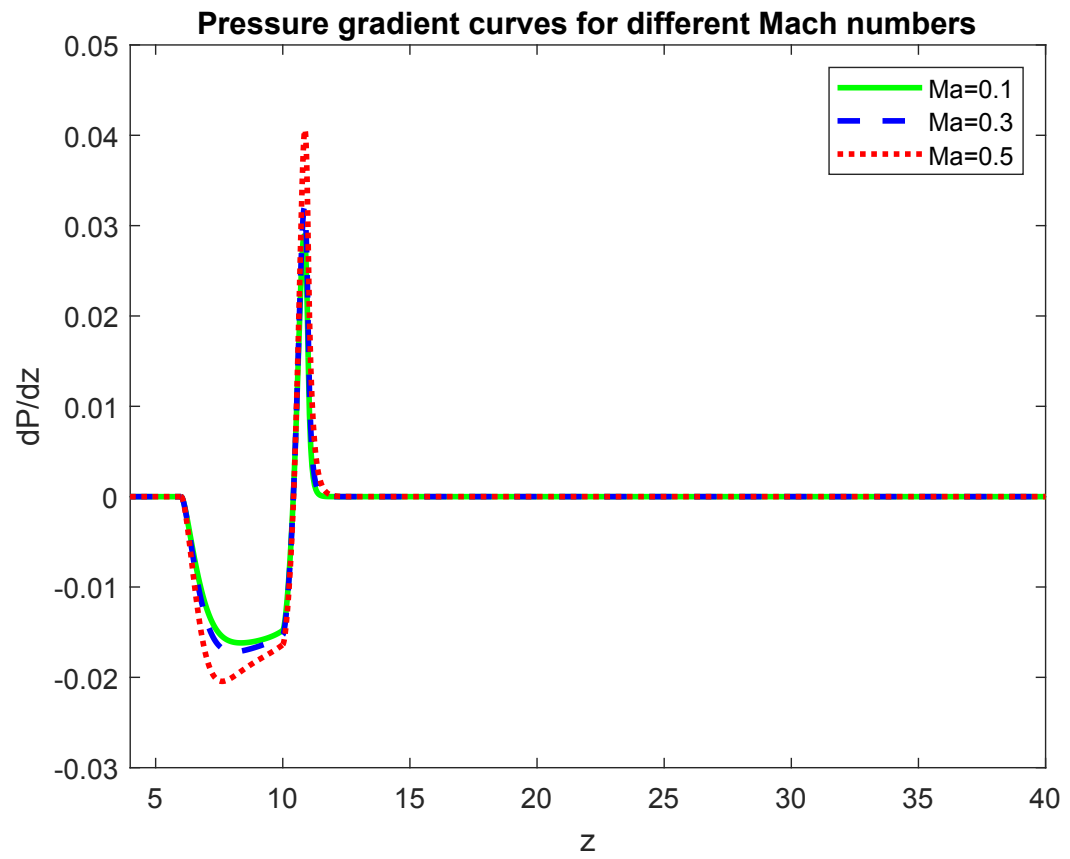

Figure 4.6: Pressure gradient curves in piecewise polynomial geometry with $w_{s}^{0}=0.04, l_{1}=4, l_{2}=1$, $R_{0}=0.1, \delta=0.05, R e_{0}=200$ for various Mach numbers 


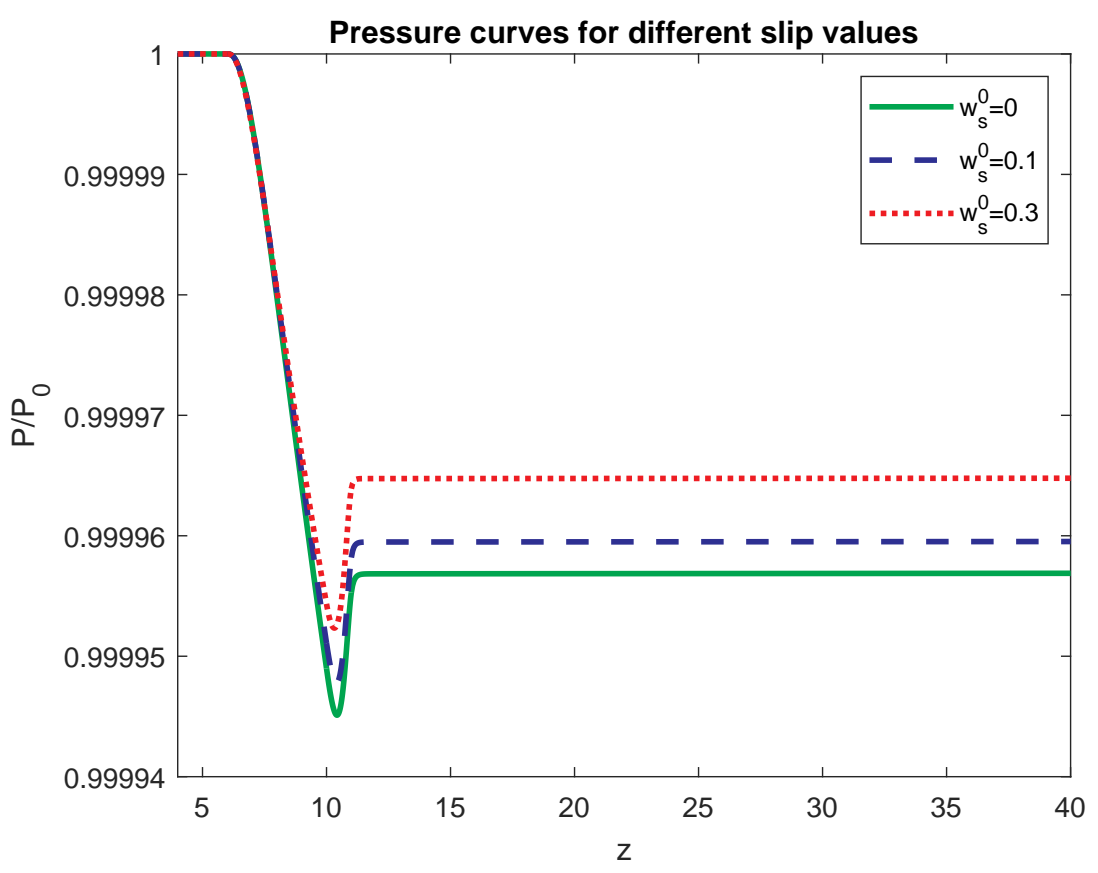

Figure 4.7: Pressure curves in piecewise polynomial geometry with $R e_{0}=200, l_{1}=4, l_{2}=1, R_{0}=0.1$, $\delta=0.05, M a_{0}=0.1$ for various slip values 


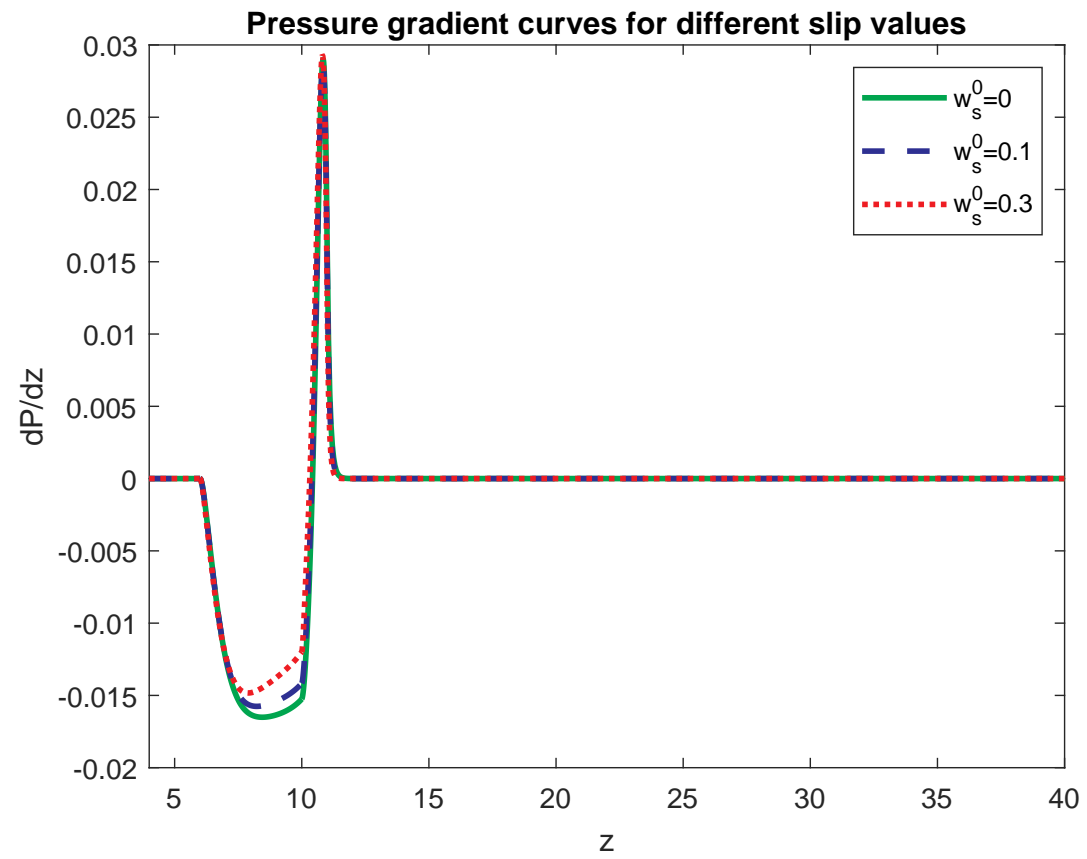

Figure 4.8: Pressure gradient curves in piecewise polynomial geometry with $R e_{0}=200, l_{1}=4, l_{2}=1$, $R_{0}=0.1, \delta=0.05, M a_{0}=0.1$ for various slip values 


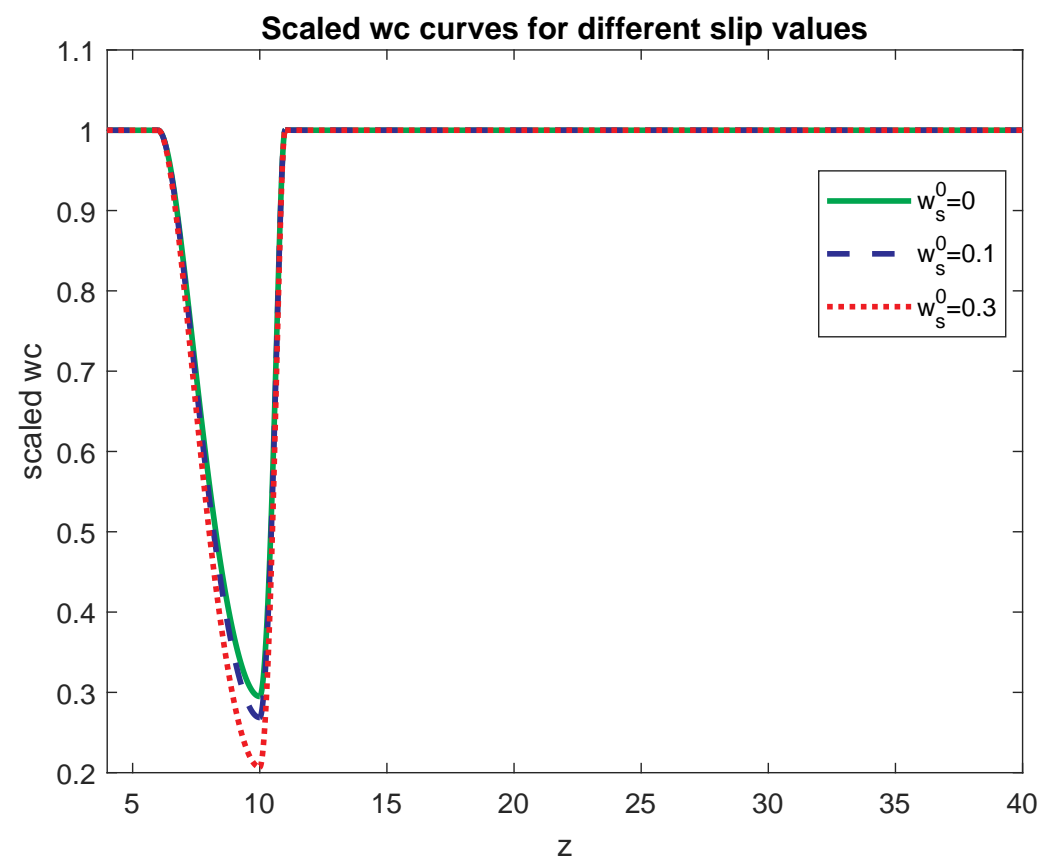

Figure 4.9: Scaled centerline velocity curves in piecewise polynomial geometry with $R e_{0}=200, l_{1}=4$, $l_{2}=1, R_{0}=0.1, \delta=0.05, M a_{0}=0.1$ for various slip values 


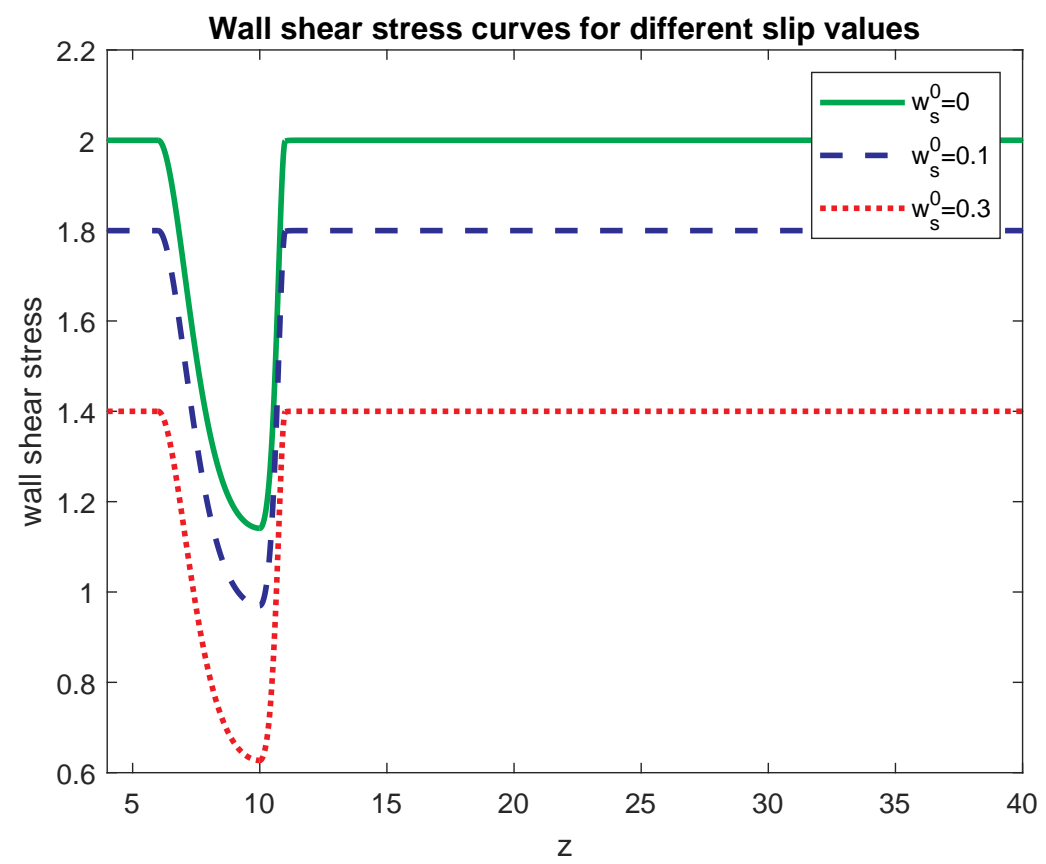

Figure 4.10: Wall shear stress curves in piece-wise polynomial geometry with $R e_{0}=200, l_{1}=4, l_{2}=1$, $R_{0}=0.1, \delta=0.05, M a_{0}=0.1$ for various slip values 


\section{Chapter 5}

\section{Conclusion and Future work}

In this Thesis the effect of blood flow through aneurysms was studied analytically. In order to study the effect, solution curves were obtained for various Reynolds numbers, compressibility (Mach numbers) and slip values. Increasing flow speeds, or considering larger vessels, would increase the 'Reynolds number' and decrease the Mach number for example.

Our analysis showed the influence of the Reynolds numbers, Mach numbers and slip on the blood flow pressure, centerline velocity, and on the wall shear stress. Scaled centerline velocity and wall shear stresses were least affected by variation in Reynolds and Mach numbers, while pressure gradient curves showed more extreme values for larger Reynolds numbers, larger Mach numbers, and smaller slip values. Reynolds number has more significant effect on the blood flow pressure as compared to the Mach numbers. Significant decrease in the pressure curve is attained for larger Reynolds numbers. The maximum decrease in the pressure curve is observed for the most compressible flows, whereas for weakly compressible or incompressible flows, minimal changes in the pressure curves are obtained.

Further investigations can be made in analyzing the solution curves by varying the degree and length of the expansion. The theoretical results presented in this Thesis are ideal for future studies to compare with particle-based flow simulations. Additionally, more complex axisymmetric flow geometries, such as a

combined stenosis and aneurysm or multiple aneurysms can be studied. A full assessment of the asymmetry of an aneurysm is also a possible extension of this work. 


\section{Appendix A}

In this Appendix, we provide the derivatives of integrals using the Leibniz rule. These results are required in the derivation of the pressure gradient equation in Chapter 2.

\section{A.1 Derivatives of integrals}

$$
\begin{aligned}
\frac{d}{d z} \int_{0}^{R} r w d r & =\left.R^{\prime}(r w)\right|_{r=R}+\int_{0}^{R} r \frac{d w}{d z} d r \\
& =\frac{R w_{s}}{\sqrt{1+R^{\prime 2}}} R^{\prime}+\int_{0}^{R} r \frac{d w}{d z} d r \\
\frac{d}{d z} \int_{0}^{R} r \rho w d r & =\left.R^{\prime}(r \rho w)\right|_{r=R}+\int_{0}^{R} r \frac{d}{d z}(\rho w) d r \\
& =\frac{R \rho w_{s}}{\sqrt{1+R^{\prime 2}}} R^{\prime}+\int_{0}^{R} r \frac{d}{d z}(\rho w) d r \\
\frac{d}{d z} \int_{0}^{R} r w^{2} d r & =\frac{R R^{\prime} w_{s}^{2}}{\left(1+R^{\prime 2}\right)}+\int_{0}^{R} r \frac{d}{d z} w^{2} d r
\end{aligned}
$$

Recall that $\rho=\rho(z)$, and $\left.w\right|_{r=R}=\frac{w_{s}}{\sqrt{1+R^{\prime 2}}}$ as per equation (2.11).

Differentiating (A.1) using $\frac{d w_{s}}{d z}=0$ gives

$$
\begin{aligned}
\frac{d^{2}}{d z^{2}} \int_{0}^{R} r w d r= & \frac{d}{d z}\left(\frac{R w_{s}}{\sqrt{1+R^{\prime 2}}} R^{\prime}\right)+\frac{d}{d z} \int_{0}^{R} r \frac{d}{d z}(w) d r \\
= & \int_{0}^{R} r \frac{d^{2} w}{d z^{2}} d r+\frac{R w_{s}}{\sqrt{1+R^{\prime 2}}} \frac{d^{2} R}{d z^{2}}+\frac{{R^{\prime}}^{2} w_{s}}{\sqrt{1+R^{\prime 2}}} \\
& -\frac{2 R R^{\prime 2} w_{s}}{\left(1+R^{\prime 2}\right)^{\frac{3}{2}}} \frac{d^{2} R}{d z^{2}} .
\end{aligned}
$$


Similarly, differentiating (A.2) gives

$$
\begin{aligned}
\frac{d^{2}}{d z^{2}} \int_{0}^{R} r \rho w d r=\frac{\rho\left(R R^{\prime \prime}+R^{\prime 2}\right) w_{s}}{\sqrt{1+R^{\prime 2}}}+\frac{2 R w_{s} R^{\prime}}{\sqrt{1+R^{\prime 2}}} \frac{d \rho}{d z} \\
-\frac{2 R \rho w_{s} R^{\prime 2} R^{\prime \prime}}{\left(1+R^{\prime 2}\right)^{3 / 2}}+\int_{0}^{R} r \frac{d^{2}}{d z^{2}}(\rho w) d r .
\end{aligned}
$$




\section{Appendix B}

\section{B.1 Constant flow rate}

In this Appendix, we show that the flow rate is constant (eg. $d Q / d z=0$ ) and develop the integral relationships given in equation (2.22) and (2.23).

Recall equation (2.20) where the flow rate is defined by

$$
Q=\pi \rho R^{2} \bar{W}=2 \pi \rho \int_{0}^{R} r w(r, z) d r .
$$

Differentiating this expression with respect to $z$ gives

$$
\begin{aligned}
\frac{d Q}{d z} & =2 \pi \int_{0}^{R} r \frac{d}{d z}(\rho w) d r+\left.2 \pi R^{\prime}(r \rho w)\right|_{r=R} \\
& =2 \pi \int_{0}^{R} r\left(-\frac{\partial}{\partial r}(\rho u)-\frac{\rho u}{r}\right) d r+\left.2 \pi R^{\prime}(r \rho w)\right|_{r=R} \\
& =-2 \pi \int_{0}^{R} \frac{\partial}{\partial r}(r \rho u) d r+\left.2 \pi R^{\prime}(r \rho w)\right|_{r=R} \\
& =-\left.2 \pi(r \rho u)\right|_{r=0} ^{r=R}+\left.2 \pi R^{\prime} R \rho w\right|_{r=R} \\
& =-\left.2 \pi R \rho u\right|_{r=R}+\left.2 \pi R^{\prime} R \rho w\right|_{r=R} \\
& =-2 \pi R \rho \frac{w_{s} R^{\prime}}{\sqrt{1+R^{\prime 2}}}+2 \pi R^{\prime} R \rho \frac{w_{s}}{\sqrt{1+R^{\prime 2}}}(\text { using }(2.11)) \\
& =0 .
\end{aligned}
$$

Thus, it follows that

$$
\frac{d Q}{d z}=0 \Rightarrow \frac{d^{n}}{d z^{n}}\left(\int_{0}^{R} r \rho w d r\right)=0
$$


and

$$
\begin{aligned}
\frac{d}{d z} \int_{0}^{R} r w d r & =\frac{d}{d z}\left(\frac{Q}{2 \pi \rho}\right) \\
& =-\frac{Q}{2 \pi} \frac{1}{\rho^{2}} \frac{d \rho}{d z} \\
& =-\frac{\pi \rho R^{2} \bar{W}}{2 \pi} \frac{1}{\rho^{2}} \frac{d \rho}{d z} \\
& =-\frac{R^{2} \bar{W}}{2 \rho} \frac{d \rho}{d z},
\end{aligned}
$$




\section{Appendix C}

In this Appendix we provide the result for the derivative of the integral of $r w^{2}$ which is required on the left-hand side of equation (2.24), obtained in [27].

$$
\begin{aligned}
& \frac{d}{d z}\left(\int_{0}^{R} r w^{2} d r\right)=\frac{61}{1580712} \frac{6 R^{5} R^{\prime}(d P / d z)^{2}+2 R^{6}(d P / d z)\left(d^{2} P / d z^{2}\right)}{\mu^{2}} \\
& +\left[\frac{631}{65863} \frac{R^{3} R^{\prime} \bar{W}}{\mu}-\frac{631}{131726} \frac{R^{4} \bar{W}(d \rho / d z)}{\rho \mu}-\frac{190}{197589} \frac{w_{s} R^{3} R^{\prime}}{\mu}\right. \\
& -\frac{61}{790356} \frac{\left(6 R^{5} R^{\prime} \rho+R^{6}(d \rho / d z)\right) g}{\mu^{2}}-\frac{899}{197589} \frac{w_{s} R^{4}}{\mu} \frac{d}{d z}\left(\frac{1}{\sqrt{1+R^{\prime 2}}}\right) \\
& \left.-\frac{899}{197589} \frac{4 w_{s} R^{3} R^{\prime}}{\mu \sqrt{1+R^{\prime 2}}}\right] \frac{d P}{d z}+\left[\frac{631}{131726} \frac{R^{4} \bar{W}}{\mu}-\frac{95}{395178} \frac{w_{s} R^{4}}{\mu}\right. \\
& \left.-\frac{61}{790356} \frac{R^{6} \rho g}{\mu^{2}}-\frac{899}{197589} \frac{w_{s} R^{4}}{\mu \sqrt{1+R^{\prime 2}}}\right] \frac{d^{2} P}{d z^{2}} \\
& +\frac{23837}{1975890}\left[\frac{2 R R^{\prime}}{\sqrt{1+R^{\prime 2}}}+R^{2} \frac{d}{d z}\left(\frac{1}{\sqrt{1+R^{\prime 2}}}\right)\right] w_{s}^{2} \\
& -\frac{154937}{395178} \rho R^{2} \bar{W} w_{s}\left[\frac{1}{\rho} \frac{d}{d z}\left(\frac{1}{\sqrt{1+R^{\prime 2}}}\right)-\frac{1}{\sqrt{1+R^{\prime 2}}} \frac{d \rho}{d z} \frac{1}{\rho^{2}}\right] \\
& +\frac{899}{197589} \frac{g w_{s}}{\mu}\left[4 R^{3} R^{\prime} \rho \frac{1}{\sqrt{1+R^{\prime 2}}}+R^{4} \frac{d \rho}{d z} \frac{1}{\sqrt{1+R^{\prime 2}}}\right. \\
& \left.+R^{4} \rho \frac{d}{d z}\left(\frac{1}{\sqrt{1+R^{\prime 2}}}\right)\right]
\end{aligned}
$$




$$
\begin{aligned}
& +\frac{5125}{395178} \frac{R^{2} \bar{W} w_{s}}{\rho} \frac{d \rho}{d z}+\frac{6610}{9409} \rho^{2} R^{4} \bar{W}^{2}\left[-\frac{2 R^{\prime}}{R^{3} \rho^{2}}-\frac{2(d \rho / d z)}{R^{2} \rho^{3}}\right] \\
& +\frac{61}{1580712} \frac{g^{2}}{\mu^{2}}\left[6 R^{5} R^{\prime} \rho^{2}+2 R^{6} \rho \frac{d \rho}{d z}\right]+\frac{298}{329315} w_{s}^{2} R R^{\prime} \\
& +\frac{95}{395178} \frac{g w_{s}}{\mu}\left[4 R^{3} R^{\prime} \rho+R^{4} \frac{d \rho}{d z}\right]-\frac{631}{65863} \frac{\rho R^{3} \bar{W} g R^{\prime}}{\mu} \\
& +\frac{26816}{141135} w_{s}^{2}\left[\frac{2 R R^{\prime}}{1+R^{\prime 2}}+\frac{2 R^{2}}{\sqrt{1+R^{\prime 2}}} \frac{d}{d z}\left(\frac{1}{\sqrt{1+R^{\prime 2}}}\right)\right]
\end{aligned}
$$




\section{References}

[1] T. Akhter and K. Rohlf. Quantifying compressibility and slip in multiparticle collision (MPC) flow through a local constriction. Entropy, 16(1):418-442, 2014.

[2] S. Bedkihal, J.C. Kumaradas, and K. Rohlf. Steady flow through a constricted cylinder by multiparticle collision dynamics. Biomechanics and modeling in mechanobiology, 12(5):929-939, 2013.

[3] A. Bit and H. Chattopadhay. Acute aneurysm is more critical than acute stenoses in blood vessels: a numerical investigation using stress markers. BioNanoScience, 8(1):329-336, 2018.

[4] H. Brunel, D. Ambard, H. Dufour, PH. Roche, V. Costalat, and F. Jourdan. Rupture limit evaluation of human cerebral aneurysms wall: Experimental study. Journal of biomechanics, 77:76-82, 2018.

[5] S. Chandra, V. Gnanaruban, F. Riveros, J.F. Rodriguez, and E.A. Finol. A methodology for the derivation of unloaded abdominal aortic aneurysm geometry with experimental validation. Journal of biomechanical engineering, 138(10):101005, 2016.

[6] K.J. Chodzyński, O.F. Eker, A.E. Vanrossomme, D.R. de Sousa, G. Coussement, L. Vanhamme, F. Dubois, A. Bonafé, B. Chopard, G. Courbebaisse, and others. Does the gravity orientation of saccular aneurysms influence hemodynamics? An experimental study with and without flow diverter stent. Journal of biomechanics, 49(16):3808-3814, 2016.

[7] JR. Darias, M. Quiroga, E. Medina, PJ. Colmenares, and R. Paredes. Simulation of suspensions in constricted geometries by dissipative particle dynamics. Molecular Simulation, 29(6-7):443-449, 2003.

[8] V. Deplano, Y. Knapp, L. Bailly, and E. Bertrand. Flow of a blood analogue fluid in a compliant abdominal aortic aneurysm model: Experimental modelling. Journal of biomechanics, 47(6):1262-1269, 2014.

[9] R. Devanathan and S. Parvathamma. Flow of micropolar fluid through a tube with stenosis. Medical and Biological Engineering and Computing, 21(4):438-445, 1983.

[10] C. Drapaca. Poiseuille flow of a non-local non-Newtonian fluid with wall slip: a first step in modeling cerebral microaneurysms. Fractal and fractional, 2(1):9, 2018.

[11] S. D'Souza. Aneurysmal subarachnoid hemorrhage. Journal of neurosurgical anesthesiology, 27(3):222, 2015. 
[12] A. Elhanafy, A. Guaily, and A. Elsaid. Numerical simulation of blood flow in abdominal aortic aneurysms: Effects of blood shear-thinning and viscoelastic properties. Mathematics and Computers in Simulation, 160:55-71, 2019.

[13] V.L. Feigin, G.JE. Rinkel, C.MM. Lawes, A. Algra, D.A. Bennett, Gilin. J.v, and C.S. Anderson. Risk factors for subarachnoid hemorrhage: an updated systematic review of epidemiological studies. Stroke, 36(12):2773-2780, 2005.

[14] F. Fillinger, M, P. Marra, S, M.L. Raghavan, and F.E. Kennedy. Prediction of rupture risk in abdominal aortic aneurysm during observation: wall stress versus diameter. Journal of vascular surgery, 37(4):724$732,2003$.

[15] J.H. Forrester and D.F. Young. Flow through a converging-diverging tube and its implications in occlusive vascular disease - i: Theoretical development. Journal of Biomechanics, 3(3):297-305, 1970.

[16] K. Jain, S. Roller, and K. Mardal. Transitional flow in intracranial aneurysms-a space and time refinement study below the Kolmogorov scales using lattice Boltzmann method. Computers \&f Fluids, 127:36-46, 2016.

[17] F. Karami, A.A. Nadooshan, L.G. Westerberg, and Y.T. Beni. Nanofluid flow in a catheterized tapered artery. Journal of Thermal Analysis and Calorimetry, 137(1):327-342, 2019.

[18] AK. Khe, AP. Chupakhin, AA. Cherevko, DV. Parshin, AL. Krivoshapkin, and K Yu. Orlov. Personalized mathematical modeling of cerebral arterial aneurysms. In 2015 International Conference on Biomedical Engineering and Computational Technologies (SIBIRCON), pages 230-232. IEEE, 2015.

[19] BV.R. Kumar and KB. Naidu. Finite element analysis of nonlinear pulsatile suspension flow dynamics in blood vessels with aneurysm. Computers in biology and medicine, 25(1):1-20, 1995.

[20] Z. Li and C. Kleinstreuer. A comparison between different asymmetric abdominal aortic aneurysm morphologies employing computational fluid-structure interaction analysis. European Journal of MechanicsB/Fluids, 26(5):615-631, 2007.

[21] S. Mukhopadhyay and GC. Layek. Analysis of blood flow through a modelled artery with an aneurysm. Applied Mathematics and Computation, 217(16):6792-6801, 2011.

[22] S. Nadeem, N.S. Akbar, A.A. Hendi, and T. Hayat. Power law fluid model for blood flow through a tapered artery with a stenosis. Applied Mathematics and Computation, 217(17):7108-7116, 2011.

[23] Y. Nubar. Blood flow, slip, and viscometry. Biophysical journal, 11(3):252-264, 1971.

[24] R. Padma, R.T. Selvi, and R. Ponalagusamy. Effects of slip and magnetic field on the pulsatile flow of a jeffrey fluid with magnetic nanoparticles in a stenosed artery. The European Physical Journal Plus, 134(5):221, 2019. 
[25] P. Paudel and K. Rohlf. Flow with slip through an aneurysm using particle-based methods. Proceedings of the 23rd Canadian Congress of Applied Mechanics (CANCAM), Vancouver, BC, Canada., pages 5-9, 2011.

[26] RN. Pralhad and DH. Schultz. Modeling of arterial stenosis and its applications to blood diseases. Mathematical biosciences, 190(2):203-220, 2004.

[27] S. Rabba and K. Rohlf. Pressure curves for compressible flows with slip through asymmetric local constrictions. J. Appl. Nonlinear Sci, 3:21-40, 2018.

[28] Y.M. Ruigrok, G.JE. Rinkel, and C. Wijmenga. Genetics of intracranial aneurysms. The lancet neurology, 4(3):179-189, 2005.

[29] KA. Vardulaki, NM. Walker, NE. Day, SW. Duffy, HA. Ashton, and RAP. Scott. Quantifying the risks of hypertension, age, sex and smoking in patients with abdominal aortic aneurysm. British journal of surgery, 87(2):195-200, 2000.

[30] L. Wang, B. Shi, and Z. Chai. A lattice Boltzmann study of the asymmetry effect on the hemodynamics in stented fusiform aneurysms. Computers 83 Mathematics with Applications, 71(1):328-348, 2016.

[31] F. Weichert, L. Walczak, D. Fisseler, T. Opfermann, M. Razzaq, R. Münster, S. Turek, I. Grunwald, C. Roth, C. Veith, and others. Simulation of intra-aneurysmal blood flow by different numerical methods. Computational and mathematical methods in medicine, 2013, 2013.

[32] MA. Xenos. An Euler-Lagrange approach for studying blood flow in an aneurysmal geometry. Proceedings of the Royal Society A: Mathematical, Physical and Engineering Sciences, 473(2199):20160774, 2017.

[33] A. Zaman, N. Ali, and O.A. Bég. Numerical simulation of unsteady micropolar hemodynamics in a tapered catheterized artery with a combination of stenosis and aneurysm. Medical $\mathbb{E}$ biological engineering Es computing, 54(9):1423-1436, 2016.

[34] A. Zaman, N. Ali, and N. Kousar. Nanoparticles (cu, tio2, al2o3) analysis on unsteady blood flow through an artery with a combination of stenosis and aneurysm. Computers 85 Mathematics with Applications, 76(9):2179-2191, 2018.

[35] J.G. Zhou. Axisymmetric lattice Boltzmann method. Physical Review E, 78(3):036701, 2008. 